\title{
University of \\ Leicester
}

\section{DEPARTMENT OF ECONOMICS}

\section{A GENERAL THEORY OF TIME DISCOUNTING: \\ THE REFERENCE-TIME THEORY OF \\ INTERTEMPORAL CHOICE}

Ali al-Nowaihi, University of Leicester, UK

Sanjit Dhami, University of Leicester, UK

Working Paper No. 08/22

July 2008 


\title{
A general theory of time discounting: The reference-time theory of intertemporal choice
}

\author{
Ali al-Nowaihi* ${ }^{*} \quad$ Sanjit Dhami ${ }^{\dagger}$
}

1 July 2008

\begin{abstract}
We develop a general theory of intertemporal choice: the reference-time theory, RT. RT is a synthesis of ideas from the generalized hyperbolic model (Loewenstein and Prelec 1992), the quasi-hyperbolic model (Phelps and Pollak 1968, Laibson 1997) and subadditivity of time discounting (Roelofsma and Read 2000, Read 2001 and Scholten and Read 2006a). These models are extended to allow for (i) reference points for time and wealth, and (ii) different discount functions for gains and losses. $\mathrm{RT}$ is able to account for all the 6 main anomalies of time discounting: gain-loss asymmetry, magnitude effect, common difference effect, delay-speedup asymmetry, apparent intransitivity of time preferences, and non-additivity of time discounting. We provide a class of utility functions compatible with RT. We show how RT can be extended to incorporate uncertainty and attribute models of intertemporal choice.

Keywords: Anomalies of the discounted utility model, Hyperbolic discounting, Prospect theory, $\gamma$-delay, $\alpha$-subadditivity, SIE value functions.

JEL Classification Codes: C60(General: Mathematical methods and programming); D91(Intertemporal consumer choice).
\end{abstract}

*Department of Economics, University of Leicester, University Road, Leicester. LE1 7RH, UK. Phone: +44-116-2522898. Fax: +44-116-2522908. E-mail: aa10@le.ac.uk.

${ }^{\dagger}$ Department of Economics, University of Leicester, University Road, Leicester. LE1 7RH, UK. Phone: +44-116-2522086. Fax: +44-116-2522908. E-mail: Sanjit.Dhami@le.ac.uk. 


\section{Introduction}

It is commonly believed that the exponential discounted utility model of intertemporal choice (henceforth, EDU) is contradicted by a relatively large body of empirical and experimental evidence; see the survey by Frederick et al. (2002). ${ }^{1}$ Moreover, it appears that these anomalies are not simply mistakes; see Frederick et al. (2002), section 4.3. If we wish to develop models that provide a better explanation of economic behavior over time, then it is imperative to take account of these anomalies. Furthermore, certain types of behavior, and several institutional features, can be explained by decision makers attempting to deal with time-inconsistency problems that arise from non-exponential discounting; see, for instance, Frederick et al. (2002), especially their section 5 titled "Alternative Models".

\subsection{Anomalies of intertemporal choice}

Loewenstein and Prelec (1992), henceforth 'LP', described the following four anomalies, all with good empirical support ${ }^{2}$ :

1. Gain-loss asymmetry. Subjects in a study by Loewenstein (1988b) were, on average, indifferent between receiving $\$ 10$ immediately and receiving $\$ 21$ one year later (an implied discount rate of $74 \%$ per annum ${ }^{3}$ ). They were also indifferent between loosing $\$ 10$ immediately and losing $\$ 15$ dollars one year later (an implied discount rate of $40.5 \%$ per annum). Note that this is a refutation of neoclassical economics for two reasons. First, the implied discount rates are different, second, they are both too high (even allowing for capital market imperfections and liquidity constraints).

\footnotetext{
${ }^{1}$ Critical reviews can also be found in Andersen et al. (2007), Fudenberg and Levine (2006), Halevy (2007), Manzini and Mariotti (2006), Noor (2008a,b), Ok and Masatlioglu (2007), Read (2001), Roelofsma and Read (2000), Rubinstein (2003) and Scholten and Read (2006a, 2006b). For a recent overview, see Manzini and Mariotti (2008).

${ }^{2}$ We use the same terminology as LP. However, gain-loss asymmetry is also known as the sign effect. The common difference effect is also referred to as the delay effect or as preference reversal (we are grateful to a referee for pointing this out). However, 'preference reversal' is also used to describe (for example) the following situation: A consumer strictly prefers bundle $x$ to bundle $y$. But when a new bundle, $z$, is introduced, the consumer now strictly prefers $y$ to $x$. The latter phenomenon is a framing effect and leads to violation of independence. The 'common difference effect' need not be due to framing nor need it lead to a violation of independence. See, for example, Tversky and Kahneman (1991), Slovic (1991) and Kahneman, Ritov and Schkade (1999). On this point, see also Manzini and Mariotti (2008, subsection $3.1)$.

${ }^{3}$ The estimated discount factor $D^{e}$ is the ratio of current to future reward times the ratio of marginal utilities. Assuming that the marginal utilities are approximately the same, the ratio of rewards is simply used to approximate $D^{e}$. Thus, in this case, $D^{e}=\frac{10}{21}=0.47619$. Assuming continuous compounding, $D^{e} \approx D=e^{-\theta}$, where $\theta$ is the discount rate. Taking logs on both sides, $\theta=-\ln D^{e}$, which in this case is $-\ln (0.47619)=0.74194$, as claimed. The same method is used to report the other discount rates in experiments, below. For critiques of this approach, see Noor $(2008 \mathrm{a}, \mathrm{b})$.
} 
2. Magnitude effect. Thaler (1981) reported that subjects were, on average, indifferent between receiving $\$ 15$ immediately and $\$ 60$ one year later (an implied discount rate of $139 \%$ per annum). They were also indifferent between receiving $\$ 3000$ immediately and receiving $\$ 4000$ one year later (an implied discount rate of $29 \%$ per annum). This is a refutation because the implied discount rate is magnitude dependent and is too high.

3. Common difference effect. Thaler (1981): A person might prefer one apple today to two apples tomorrow (an implied real discount rate of $25300 \%$ per annum), but at the same time prefer two apples in 51 days to one apple in 50 days (there is no evidence that this is due to an expected change in the real discount rate). ${ }^{4}$

4. Delay-speedup asymmetry. Loewenstein (1988a) reported that, in general, the amount required to compensate for delay in receiving a real reward by a given interval, from $s$ to $s+t$, was two to four times greater than what the subjects were willing to sacrifice to bring consumption forward from $s+t$ to $s$. (Neo)classically, these quantities should be the same.

Recent scholarship has added two further anomalies:

5. Non-additivity of time discounting. Discounting from time $t$ back to time $s$ then further back to time $r$ is not the same as discounting from time $t$ back to time $r$ in one step (Read, 2001 and Scholten and Read, 2006a).

6. Intransitivity of time preferences. The following cycles have been observed: $x$ received at time $r$ is preferred to $y$ received at time $s$ which is preferred to $z$ received at time $t$ which is preferred to $x$ received at time $r$ (Roelofsma and Read, 2000).

\subsection{Explaining the anomalies}

We briefly summarize here some attempts at explaining anomalies 1-6. These attempts will be discussed in greater depth later in the paper.

1. Generalized hyperbolic discounting (LP). LP provided the first coherent explanation of anomalies 1-4. They also provided an axiomatic derivation of their generalized hyperbolic discount function, which we shall call the LP-discount function. LP explained the magnitude effect through a value function with increasing elasticity. This makes higher magnitudes more salient. They explained gain-loss asymmetry by adopting a value function with greater elasticity for losses than for gains, which

\footnotetext{
${ }^{4}$ However, Andersen et al (2007), by jointly eliciting attitudes to risk and time, estimate much lower discount rates, of about $10.1 \%$.
} 
makes losses more salient. The common difference effect is explained in LP by the notion of declining impatience (roughly, one is more impatient as the date of the reward approaches). Hence, despite identical intervals separating two time-outcome pairs, the choice among the two depends on how close to the current period they are. However, LP cannot explain anomalies 5 and 6.

2. Quasi-hyperbolic discounting (PPL). Phelps and Pollak (1968) and Laibson (1997), 'PPL' for short, provided the very tractable quasi-hyperbolic discount function. This is also known in the literature as the $\beta-\delta$ discount function but we shall call it the PPL-discount function. It is, by far, the most popular discount function in applications (after, of course, exponential discounting). The explanation of the common difference effect in PPL is by a sudden drop in impatience at time zero (followed by constant impatience thereafter). Using the other LP assumptions, PPL can explain anomalies 1, 2 and 4 in the same way. Like LP, PPL cannot explain anomalies 5 and 6 .

3. Subadditivity and intransitivity (RRS). Roelofsma and Read (2000) provided experimental evidence for non-transitivity of time preferences. The experimental work of Read (2001) confirmed the common difference effect but rejected declining impatience in favour of constant impatience and, hence, rejected the LP (and PPL) explanation of the common difference effect in terms of declining impatience. Read (2001) and Scholten and Read (2006a) found experimental evidence for subadditivity and introduced the concept of an interval discount function ${ }^{5}$. Furthermore, based on empirical evidence, Scholten and Read (2006a) developed a specific interval discount function, which we shall call the $R S$-discount function. The RS-discount function (depending on parameter values) can explain the common difference effect as due to either declining impatience, subadditivity or a combination of both. We shall refer to this work collectively as 'RRS'.

4. Attribute based models. Another possibility is to suppose that the time-outcome pairs which are to be compared consist of two natural attributes: time of delivery and monetary value. One can then consider the tradeoff between the two attributes in determining which time-money pair will be chosen.

4.1 Rubinstein (2003) advocated the use of a similarity relationship. If a larger reward is available earlier, then this is always preferred to a smaller reward available later. However, if the choice is between a smaller-earlier pair and a

\footnotetext{
${ }^{5}$ An interval discount function, $D(r, t), 0 \leq r \leq t$, discounts a quantity from time $t$ back to time $r$. If $D$ is additive (and only if $D$ is additive), then $D(r, t)=D(r, s) D(s, t)$, for all $s, r \leq s \leq t$.
} 
larger-later pair, then there is a conflict between the attribute of money and that of time. In this case, choice is lexicographic. If either the time or the money dimension is 'similar' then it is ignored and the two pairs are then compared across the remaining dimension and a sensible choice made (e.g. more money or earlier time). Failing this, if there is no similarity in either dimension, then no decision can be made. Thus the preference relationship in Rubinstein (2003) is incomplete.

4.2 Manzini and Mariotti (2006), in their 'theory of vague time preferences', develop an attribute model that can explain the common difference effect. Manzini and Mariotti propose three criteria to choose between a smaller amount of money delivered sooner (SS) and a larger amount of money delivered later (LL). The primary criterion is to choose whichever has the highest present utility value. If the two present values are not 'significantly' different, then the subject chooses the one with the highest monetary value (secondary criteria). If they have the same monetary values, so that the secondary criterion fails, then the subject behaves according to the third criterion: "choose the outcome that is delivered sooner'. If all three criteria fail, then the subject is indifferent. Thus, Manzini and Mariotti achieve a complete, though intransitive, ordering. In particular, indifference here is not an equivalence relationship. On the other hand, the experimental results of Roelofsma and Read (2000) supported 'sooner is better than larger' against 'larger is better than sooner'. However, if the order of the secondary criteria is reversed, so that sooner is better than larger (in agreement with the experimental results of Roelofsma and Reed, 2000), then we would not get a common difference effect. However, whether Manzini and Mariotti's explanation of the common difference effect is acceptable or not, to us the main contribution of their paper lies in the use of primary and secondary criteria. This appears to us to be a more accurate description of actual decision making then the assumption of a single criterion (see subsection 5.3, below).

4.3 Scholten and Read (2006b) present a critique of the psychological basis for discounting models (including their own). They develop an attribute model based on firmer psychological foundations. In subsection 5.1, we argue that their tradeoff model is equivalent to a discounted utility model that is a generalization of their model (Scholten and Read, 2006a). If this is accepted, then their tradeoff model lends further support to their own discount function, the RSdiscount function.

5. Uncertainty and exponential discounting. Under uncertainty the common difference effect never arises when we use expected utility with exponential discounting. Hence, 
it is quite possible that the experimental finding of the common difference effect is a rejection of expected utility rather than exponential discounting. Halevy (2007) shows that when non-expected utility is combined with exponential discounting, the theory is consistent with the presence of a common difference effect, provided uncertainty is present but sufficiently small (see subsection 5.2, below).

6. Intransitivity and relative discounting. Ok and Masatlioglu (2007) assume neither transitivity nor additivity. In its present formulation, it cannot account for either gain-loss asymmetry or delay-speedup asymmetry. But a more serious problem is the lack of transitivity. Thus it appears that it will be hard to work with this theory, as the authors themselves explain. On the other hand, these problems can all be resolved in the special case of a transitive preference relation. But then their model becomes additive. In this case, Ok and Masatlioglu would reduce to a standard discounting model (see subsection 5.4, below).

\subsection{Towards a general theory of intertemporal choice: The reference-time the- ory (RT)}

Within the class of time preference models which are separable in time and outcomes (also known as delay-discounting models), what should a general theory of time preference aspire to? We suggest two desirable elements. First, it should be able to explain anomalies 1-6. Second, it should provide a framework that can incorporate recent developments in time discounting such as uncertainty (Halevy, 2007) and attribute models (Manzini and Mariotti, 2006 and Scholten and Read, 2006b).

The aim of this paper is to develop a theory of intertemporal choice that incorporates the two desirable elements mentioned above. We call this theory the reference-time theory of intertemporal choice, 'RT' for short. It is a synthesis of three earlier seminal works, namely, LP, PPL and RRS. In a nutshell, RT is basically LP extended to allow for nonadditive time discounting by incorporating a reference point for time. ${ }^{6}$ We explain the anomalies 1-6 as follows.

1. Like LP, we explain the magnitude effect (anomaly 2) by assuming that the elasticity of the value function is increasing. However, in section 3, we show that several popular classes of utility functions violate this condition. These includes CARA (constant absolute risk aversion), CRRA (constant relative risk aversion), HARA (hyperbolic absolute risk aversion), logarithmic and quadratic. We develop a scheme for generating value functions that exhibit increasing elasticity, as required to explain

\footnotetext{
${ }^{6}$ The first version of this paper was written entirely within the LP framework. We are grateful to a referee for suggesting we attempt to extend it to include more recent development, in particular nonadditivity and intransitivity.
} 
the magnitude effect. The simplest class that has this property we call the class of simple increasing elasticity value functions (SIE). Each member of this class is formed by a product of a HARA function and a CRRA function and, therefore, is quite tractable. We provide a class of utility functions compatible with any theory where preferences are separable in time and outcomes. This includes RT theory, as well as LP, PPL, RRS, Halevy (2007) and Manzini and Mariotti (2006) (sections 3, 4 and 5 , below).

2. LP explained the gain-loss asymmetry (anomaly 1) by assuming that the elasticity of the value function for losses exceeds the elasticity for gains. This allows them to use the same discount function for gains and losses, in agreement with the strict separability of time and outcomes. The downside of their approach is that the coefficient of loss aversion is then variable, which contradicts the empirical evidence. In Example 4, section 4, below, we explore the implications of having the same discount function for gains and losses, as in LP. We show that, as time goes to infinity, the coefficient of loss aversion also goes to infinity. This is in contrast to the empirical findings of a constant coefficient of loss aversion, approximately equal to 2.25 (Tversky and Kahneman, 1991, 1992). For this reason, we assume a constant coefficient of loss aversion. This, in turn, forces us to adopt different discount functions for gains and losses to explain anomaly $1 .^{7}$

3. LP provided an axiomatic derivation of their generalized hyperbolic discount function (which we call the LP-discount function). For this, they added the extra assumption of linear delay to that of the common difference effect. While there is considerable empirical evidence for the common difference effect, the assumption of linear delay is added purely for convenience. We extend the LP derivation as follows. At the most general level, which requires neither linear delay nor the common difference effect, we have our Representation Theorem 2 (Proposition 12, below). We introduce a weaker notion of subadditivity, which we call $\alpha$-subadditivity (Definition 12, below). According to our Characterization Theorem 4 (Proposition 21, below), preferences exhibit the common difference effect if, and only if, $\alpha$-subadditivity holds. We also introduce a generalization of the concept of linear delay of LP. We call this $\gamma$-delay. Our Proposition 23, below, then shows that $\gamma$-delay implies the common difference effect. Imposing additivity, as well as $\gamma$-delay, gives our Proposition 24, below. The special case of the latter with $\gamma=1$ gives the LP-discount function. Our more general approach also allows us to derive the RS-discount function (Proposition 25, below). In particular, as with RRS, we can explain the common difference effect

\footnotetext{
${ }^{7}$ We are grateful for the comments of a critical referee, which helped us clarify these issues.
} 
(anomaly 3) as due to either declining impatience, subadditivity or a combination of both. However, our approach is more general, as RT can also explain the common difference effect as due to the presence of a small amount of irremovable uncertainty, as in Halevy (2007), or as a consequence of multiple decision criteria, as in Manzini and Mariotti (2006) (see section 5, below). Thus, RT can accommodate all the known explanations of the common difference effect. In the spirit of RRS, we leave it to empirical evidence to select the correct explanation.

4. We show the delay-speedup asymmetry (anomaly 4) follows from our other assumptions (see Proposition 30, below).

5. We follow LP in adopting prospect theory (Kahneman and Tversky, 1979 and Tversky and Kahneman, 1992) as our underlying decision theory. But, in addition to a reference point for wealth, we introduce a reference point for time. If preferences are additive and if the discount function for gains is the same as that for losses, as in LP, then the choice of the reference point for time is immaterial (Proposition 7, below). However, if preferences are non-additive, then the choice of the reference point for time matters. Thus, we can accommodate non-additive preferences (anomaly 5) by having a reference point for time. A consequence is that all our discount functions are interval discount functions, as in RRS.

6. Given a reference point for wealth, $w_{0}$, and a reference point for time, $r$, our preferences are complete and transitive (subsection 2.3, below). Thus they may be called conditionally complete and conditionally transitive (conditional on $w_{0}$ and $r$ ). We explain observed intransitivity as due to a change in the reference point for time (see subsections 2.4 and 2.8, below). This is in contrast to Ok and Masatlioglu (subsection 5.4, below), where preferences are complete but intransitive (in our terminology we may describe such preferences as unconditionally complete but not even conditionally transitive). Thus the relative-discounting theory Ok and Masatlioglu and the reference-time theory of this paper are not compatible and neither is a special case of the other.

To summarize, the theory presented in this paper (reference-time theory or RT) can explain anomalies 1-6 (section, 4, below) and can be extended to incorporate uncertainty, as in Halevy (2007), and the attribute models of Manzini and Mariotti (2006) and Scholten and Read (2006b) (section 5, below).

All proofs are contained in the appendix. 


\section{A reference-time theory of intertemporal choice (RT)}

This section is structured as follows. We first discuss the anomalies of intertemporal choice (subsections 2.1). Next we outline prospect theory (subsection 2.2), which is an essential building block of RT. We define preferences for RT as early as possible (subsection 2.3). This is followed by further essential material on discount functions, additivity, impatience, intransitivity and the common difference effect (subsections 2.5, 2.6, 2.7, 2.8 and 2.9). The main technical machinery: representation, extension and characterization theorems are developed in subsections 2.10, 2.11 and 2.12). Finally, in subsection 2.13 we are in a position to extend LP to allow for non-additive time discounting by incorporating a reference point for time (and also separate discount functions for gains and losses).

\subsection{Anomalies of intertemporal choice: Refutations of the exponentially dis- counted utility model or of neoclassical economics more generally?}

To fix ideas, we start with a simple neoclassical example.

Example 1 (A neoclassical example): Consider a single consumer who lives for two periods. He has exogenous incomes $I_{1}$ and $I_{2}$ in periods 1 and 2, respectively. He faces the exogenous fixed real interest rate, $r$, per period, compound continuously. Let $c_{1}$ and $c_{2}$ be real consumption in periods 1 and 2, respectively. Then the consumer's intertemporal budget constraint is

$$
c_{1}+e^{-r} c_{2}=I_{1}+e^{-r} I_{2} .
$$

Let the consumer's utility function be

$$
U\left(c_{1}, c_{2}\right)
$$

The consumer's problem is then to maximize $U\left(c_{1}, c_{2}\right)$ subject to the budget constraint (2.1) and non-negativity constrains:

$$
c_{1} \geq 0, c_{2} \geq 0
$$

Note well: the income stream $\left(I_{1}, I_{2}\right)$ is relevant to the decision problem only through its present value, $I_{1}+e^{-r} I_{2}$, discounted by the real interest rate available to the consumer, $r$. In particular, if a subject is offered a choice between real monetary rewards, $x$ in period 1 and $y$ in period 2, then the subject should choose $x$ over $y$ if, and only if, $x>e^{-r} y$ (and choose $y$ over $x$ if, and only if, $x<e^{-r} y$ ). So, if a subject exhibits behavior inconsistent with this rule, that behavior would constitute a refutation of neoclassical economics, and not just a specific functional form for $U$ (as no such assumption has been made above). For example, if subjects exhibit a clear preference for increasing income streams over 
constant or declining income streams, all with the same present value, that behavior would constitute a refutation of neoclassical economics, and not just the exponentially discounted form. The same can be said about the anomalies reported in subsection 1.1, above. Each one of them is a refutation of neoclassical economics, not just the exponentially discounted utility model as is usually, but mistakenly, claimed.

Therefore, each attempt at explaining anomalies of intertemporal choice (as far as we know) involves some element of behavioral economics. The mental accounting literature (Thaler, 1999) argues that separate income streams might not fully be integrated. LP (and this paper) use prospect theory (Kahneman and Tversky, 1979 and Tversky and Kahneman, 1992) as the underlying decision theory. Scholten and Read (2006b) and Manzini and Mariotti (2006) use attribute models, Halevy (2007) uses non-linear transformation of probabilities and Ok and Masatlioglu (2007) assume non-transitive preferences.

For further discussion of these issues, see Manzini and Mariotti (2008) and Frederick et al. (2002), sections 3.1 "Integration of new alternatives with existing plans", 5.3.3 "Mentalaccounting models", 5.3.4 "Choice bracketing" and 6.1.2 "Intertemporal arbitrage".

\subsection{Prospect theory}

We follow LP in using prospect theory (Kahneman and Tversky, 1979, and Tversky and Kahneman, 1992) rather than standard utility theory. Prospect theory distinguishes between two phases of decision making: editing and evaluation.

In the editing phase, a decision maker simplifies a real world problem to make it amenable to formal analysis and reduce the associated cognitive load. As part of the editing phase a reference point is chosen to which outcomes are to be compared.

In the evaluation phase, a value (a real number) is attached to each feasible action by the decision maker. The action with highest value is chosen. The function, $v$, that assigns values to actions in prospect theory is called the value function, it is the analogue of the indirect utility function of standard utility theory. In standard utility theory carriers of utility are the outcomes of actions. But in prospect theory carriers of utility are deviations in outcomes from the reference point. In general, the action chosen in the evaluation phase will depend on the reference point chosen in the editing phase.

The value function, $v$, in prospect theory has four main properties: reference dependence, monotonicity, declining sensitivity, loss aversion. Furthermore, in prospect theory, there is non-linear transformation of probabilities. There is good empirical support for these feature; see, for instance, Kahneman and Tversky (2000).

We take $v$ to be the value function introduced by Kahneman and Tversky (1979). Thus 
$v$ satisfies:

$v:(-\infty, \infty) \rightarrow(-\infty, \infty)$ is continuous, strictly increasing (monotonicity).

$v(0)=0$ (reference dependence) and is twice differentiable except at 0 .

For $x>0:-v(-x)>v(x)$ (loss aversion).

Following LP, we define the elasticity of the value function as follows.

Definition 1 : The elasticity of $v$ is

$$
\epsilon_{v}(x)=\frac{x}{v} \frac{d v}{d x}, x \neq 0
$$

\subsection{Preferences}

We consider a decision maker who, at time $t_{0}$, takes an action that results in the level of wealth $w_{i}$ at time $t_{i}, i=1,2, \ldots, n$, where

$$
t_{0} \leq r \leq t_{1}<\ldots<t_{n}
$$

Time $r$ is the reference time: the time back to which all values are to be discounted. We can choose any moment of time as time zero and measure all other times relative to it. We choose to set $t_{0}=0$, i.e., the time a decision is made is always time $t=0$. If it is desired to set $t_{0} \neq 0$, then simply replace all times, $t$, below, with $t-t_{0}$. The decision maker's intertemporal utility function is given by:

$$
V_{r}\left(\left(x_{1}, t_{1}\right),\left(x_{2}, t_{2}\right), \ldots,\left(x_{n}, t_{n}\right)\right)=\sum_{i=1}^{n} v\left(x_{i}\right) D_{ \pm}\left(r, t_{i}\right)
$$

where $v$ is the value function introduced by Kahneman and Tversky (1979). $x_{i}=w_{i}-w_{0}$ is the difference between the wealth level, $w_{i}$, at time, $t_{i}$, and the reference level for wealth,

$w_{0} . D_{ \pm}\left(r, t_{i}\right)$ is the discount function, it discounts $v\left(x_{i}\right)$ from time, $t_{i}$, back to the reference time, $r . D_{ \pm}\left(r, t_{i}\right)=D_{+}\left(r, t_{i}\right)$ if $x_{i} \geq 0$ and $D_{ \pm}\left(r, t_{i}\right)=D_{-}\left(r, t_{i}\right)$ if $x_{i}<0$.

More formally, we assume that, for each $\left(w_{0}, r\right) \in(-\infty, \infty) \times[0, \infty)$, the decision maker has a complete transitive preference relation, $\preceq_{w_{0}, r}$ on $(-\infty, \infty) \times[r, \infty)$. We think of $w_{0}$ as the reference point for wealth and $r$ the reference point for time. If $(w, t) \in$ $(-\infty, \infty) \times[r, \infty)$, with $w \geq w_{0}$, we say that $(w, t)$ is an outcome in the domain of gains. If $(w, t) \in(-\infty, \infty) \times[r, \infty)$, with $w \leq w_{0}$, we say that $(w, t)$ is an outcome in the domain of losses. We assume that $\preceq_{w_{0}, r}$ is represented by a utility function $v\left(w-w_{0}\right) D_{ \pm}(r, t)$. Thus $\left(w_{1}, t_{1}\right) \preceq_{w_{0}, r}\left(w_{2}, t_{2}\right)$ if, and only if, $v\left(w_{1}-w_{0}\right) D_{ \pm}\left(r, t_{1}\right) \leq v\left(w_{2}-w_{0}\right) D_{ \pm}\left(r, t_{2}\right)$. 
Using (2.9), we extend $\preceq_{w_{0}, r}$ to a complete transitive preference relation on sequences from $(-\infty, \infty) \times[r, \infty)$, as follows ${ }^{8}$ :

$$
\begin{aligned}
& \left(\left(x_{1}, s_{1}\right),\left(x_{2}, s_{2}\right), \ldots,\left(x_{m}, s_{m}\right)\right) \preceq_{w_{0}, r}\left(\left(y_{1}, t_{1}\right),\left(y_{2}, t_{2}\right), \ldots,\left(y_{n}, t_{n}\right)\right) \\
& \Leftrightarrow V_{r}\left(\left(x_{1}, s_{1}\right),\left(x_{2}, s_{2}\right), \ldots,\left(x_{m}, s_{m}\right)\right) \leq V_{r}\left(\left(y_{1}, t_{1}\right),\left(y_{2}, t_{2}\right), \ldots,\left(y_{n}, t_{n}\right)\right)
\end{aligned}
$$

We depart from LP in the following ways. LP have a reference point for wealth but not for time. We have a reference point for wealth, $w_{0}$, and a reference point for time, $r$. LP assume the same discount function for gains and losses. We allow the discount function for gains to be different from that for losses. LP implicitly assume that the discount function is additive (Definition 5, below). We allow the discount function to be non-additive, to accommodate the empirical evidence of RRS. If the discount function is additive and is the same for gains and losses (as in LP), then the choice of the reference point for time is irrelevant, since $(x, s) \preceq_{w_{0}, r}(y, t)$ if, and only if, $(x, s) \preceq_{w_{0}, 0}(y, t)$ (Proposition 7 , below). However, if the discount function is non-additive, then the choice of the reference point for time matters. We use this to explain (apparent) intransitivity as a framing effect due to a change in the reference point for time.

\subsection{Determination of the reference point for time}

Let $S$ be a non-empty set of sequences from $(-\infty, \infty) \times[0, \infty)$. Suppose a decision maker is interested in comparing the members of $S$. For example, for the purpose of choosing the optimal member (if $S$ is compact). For this he needs a reference point for time. Let $T$ be the set of times involved, i.e.,

$$
T=\left\{t \in[0, \infty): t=t_{i} \text { for some sequence }\left\{\left(x_{1}, t_{1}\right),\left(x_{2}, t_{2}\right), \ldots,\left(x_{i}, t_{i}\right), \ldots\right\} \text { in } S\right\} \text {. }
$$

Since $T$ is bounded below (by 0 ) and non-empty, it follows that $T$ has a greatest lower bound, $r$. We make the following tentative assumption:

A0 Reference time. Given $S, T, r$, as described just above, we assume that the decision maker takes $r$ as the reference point for time.

For example, if a decision maker wants to compare $x$ received at time $s$ with $y$ received at time $t, s \leq t$, then $S$ consists of just two sequences, each with just one element: $S=\{(x, s),(y, t)\}$ and $T=\{s, t\}$. Thus A0 implies that $r=s$. If $v(x)<v(y) D(s, t)$ then the decision maker chooses $(y, t)$ over $(x, s)$.

A0 does not have the status of the LP assumptions A1-A4, introduced in subsection 2.13, below. While there is considerable, though debated, empirical evidence for A1-A4, A0 should be regarded as a tentative assumption, whose implications are to be explored.

\footnotetext{
${ }^{8}$ This also holds for infinite sequences, provided the sum in (2.9) is convergent.
} 
We will only use A0 in subsections 2.8 and 2.13. In subsection 2.8, we use A0 to explain (apparent) intransitivity as due to a shift in the reference point for time. In subsection 2.13, we use A0 to prove that assumption A4 (Delay-speedup asymmetry) follows from the other assumptions (Proposition 30).

\subsection{Discount functions}

The five discount functions that will be important for this paper are:

$$
\begin{gathered}
\text { Exponential: } D(r, t)=e^{-\beta(t-r)}, \beta>0 . \\
\text { PPL: } D(r, t)=\left\{\begin{array}{ccc}
1 & \text { when } & r=t=0 \\
e^{-(\delta+\beta t)} & \text { when } & r=0, t>0 \\
e^{-\beta(t-r)} & \text { when } & 0<r \leq t
\end{array}\right\} ; \beta>0, \delta>0 . \\
\text { LP: } D(r, t)=\left(\frac{1+\alpha t}{1+\alpha r}\right)^{-\frac{\beta}{\alpha}}, t \geq 0, r \geq 0, \alpha>0, \beta>0 .
\end{gathered}
$$

$\mathbf{R S}: D(r, t)=\left[1+\alpha\left(t^{\tau}-r^{\tau}\right)^{\rho}\right]^{-\frac{\beta}{\alpha}}, 0 \leq r \leq t, \alpha>0, \beta>0, \rho>0, \tau>0$.

generalized RS: $\quad D(r, t)=e^{-Q[w(t)-w(r)]}, 0 \leq r \leq t$,

$$
\begin{aligned}
& Q:[0, \infty) \stackrel{\text { onto }}{\longrightarrow}[0, \infty) \text { is strictly increasing, } \\
& w:[0, \infty) \stackrel{\text { onto }}{\longrightarrow}[0, \infty) \text { is strictly increasing. }
\end{aligned}
$$

The exponential discount function (2.12) was introduced by Samuelson (1937). Aside from its tractability, the main attraction of EDU is that it leads to time-consistent choices. If the plan $\left(x_{1}, t_{1}\right),\left(x_{2}, t_{2}\right), \ldots,\left(x_{n}, t_{n}\right)$ is optimal at time 0 , then at time $t_{k}$ the plan $\left(x_{k+1}, t_{k+1}\right),\left(x_{k+2}, t_{k+2}\right), \ldots,\left(x_{n}, t_{n}\right)$ is also optimal. But this may no longer be true for more general specifications of the discount function.

The $\beta \delta$ (or quasi-hyperbolic) discount function (2.13) was proposed by Phelps and Pollak (1968) and Laibson (1997). Its popularity in applied work is second only to EDU. ${ }^{9}$ The generalized hyperbolic discount function (2.14) was proposed by Loewenstein and Prelec (1992). For the special case, $\alpha=\beta$, (2.14) it reduces to the hyperbolic discount function. These three discount functions are additive (Definition 3, below). They can account for the common difference effect through declining impatience (Definition 6, below) but they cannot account for either non-additivity or intransitivity.

The interval discount function (2.15) was introduced by Scholten and Read (2006a). It can account for both non-additivity and intransitivity. It can account for the common difference effect though declining impatience, subadditivity or a combination of both (subsections 2.8 and 2.9, below).

\footnotetext{
${ }^{9}$ It can be given the following psychological foundation. The decision maker essentially uses exponential discounting. But in the short run is overcome by visceral influences such as temptation or procrastination.
} 
In subsection 5.1, below, we shall show that the attribute model of Scholten and Read (2006b) is equivalent to a discounted utility model with the discount function (2.16), which is a generalization of their RS-discount function (2.15).

Note that (2.14) approaches (2.12) as $\alpha \rightarrow 0$. In general, neither of (2.14) or (2.15) is a special case of the other. However, for $r=0$ (and only for $r=0$ ), (2.15) reduces to (2.14) when $\rho=\tau=1$. Scholten and Read (2006a) report incorrectly that the LP-discount function is a special case of the RS-discount function. One needs to restrict $r=0$ (in addition to $\rho=\tau=1$ ) in order to generate the LP from the RS-discount function. While $\rho, \tau$ are parameters, $r$ is a variable. Hence, neither discount function is a special case of the other.

We now give a formal definition of a discount function. This will be the first (and, of course, the most important) of five functions we will introduce (the others are: the generating function, the delay function, the seed function and the extension function).

Definition 2 (Discount functions): Let

$$
\Delta=\{(r, t) \in \mathbb{R} \times \mathbb{R}: 0 \leq r \leq t\} .
$$

A discount function is a mapping, $D: \Delta \rightarrow(0,1]$, satisfying:

(i) For each $r \in[0, \infty), D(r, t)$ is a strictly decreasing function of $t \in[r, \infty)$ into $(0,1]$ with $D(r, r)=1$.

(ii) For each $t \in[0, \infty), D(r, t)$ is a strictly increasing function of $r \in[0, t]$ into $(0,1]$.

Furthermore, if $D$ satisfies (i) with 'into' replaced with 'onto', then we call $D$ a continuous discount function.

Our terminology suggests that a continuous discount function is continuous. That this is partly true, is established by the following Proposition.

Proposition 1 : A continuous discount function, $D(r, t)$, is continuous in $t$.

Proposition 2 : Each of (2.12), (2.14) and (2.15) is a continuous discount function in the sense of Definition 2. However, (2.13) is a discount function but not a continuous discount function.

The reason that $(2.13)$ fails to be a continuous discount function is that $\lim _{t \rightarrow 0+} D(0, t)=$ $e^{-\delta}<1=D(0,0)$.

From (2.14) and (2.15) we see that the restrictions $r \geq 0$ and $t \geq 0$ are needed. From (2.15) we see that the further restriction $r \leq t$ is needed. ${ }^{10}$ From (2.12) we see that the 'into' in Definition 2(ii) cannot be strengthened to 'onto'.

\footnotetext{
${ }^{10}$ One alternative is to define $D(t, s)$ to be $1 / D(s, t)$. But we do not know if people, when compounding forward, use the inverse of discount function (as they should, from a normative point of view). Fortunately, we have no need to resolve these issues in this paper.
} 
Proposition 3 (Time sensitivity): Let $D$ be a continuous discount function. Suppose $r \geq 0$. If $0<x \leq y$, or if $y \leq x<0$, then $v(x)=v(y) D(r, t)$ for some $t \in[r, \infty) .{ }^{11}$

Proposition 4 (Existence of present values): Let $D$ be a discount function. Let $r \leq t$. Let $y \geq 0(y \leq 0)$. Then, for some $x, 0 \leq x \leq y(y \leq x \leq 0), v(x)=v(y) D(r, t) .{ }^{12}$

\subsection{Additivity}

We now define additivity and related concepts.

Definition 3 (Additivity): A discount function, $D(r, t)$, is

$$
\begin{gathered}
\text { additive if } D(r, s) D(s, t)=D(r, t), \text { for } r \leq s \leq t, \\
\text { subadditive if } D(r, s) D(s, t)<D(r, t) \text {, for } r<s<t \text {, } \\
\text { superadditive if } D(r, s) D(s, t)>D(r, t) \text {, for } r<s<t \text {. }
\end{gathered}
$$

Additivity (2.18) implies that discounting a quantity from time $t$ back to time $s$ and then further back to time $r$ is the same as discounting that quantity from time $t$ back to time $r$ in one step.

To aid further development, we define a generating function, whose interpretation will become apparent from Proposition 5 that follows the definition.

Definition 4 (The generating function): Let $\varphi:[0, \infty) \rightarrow(0,1]$ be a strictly decreasing function with $\varphi(0)=1$. Then we call $\varphi$ a generating function. If, in addition, $\varphi$ is onto, we call $\varphi$ a continuous generating function.

A 'continuous generating function' is continuous. The proof is the same as that of Proposition 1 and, therefore, will be omitted.

Proposition 5 (Additive extension theorem):

(a) Let $D(r, t)=[\varphi(r)]^{-1} \varphi(t)$ for some strictly decreasing real valued function, $\varphi$ : $[0, \infty) \rightarrow(0,1]$. Then the following hold:

(i) $D$ is an additive discount function.

(ii) If $\varphi(0)=1$ (so that $\varphi$ is a generating function), then $D(0, t)=\varphi(t)$.

(iii) If $\varphi$ is onto (so that $\varphi$ is a continuous generating function), then $D$ is an additive continuous discount function and $D(0, t)=\varphi(t)$.

(b) Let $D$ be an additive discount function. Then the following hold:

\footnotetext{
${ }^{11}$ We have chosen the phrase 'time sensitivity' to conform with the terminology of OM, Axiom A1, p219, and Claim 3, p235.

${ }^{12} \mathrm{We}$ are grateful to a referee for pointing out that Proposition 4 follows from (2.4), (2.5) and Definition 2 ; and for suggesting the name 'existence of a present value'.
} 
(i) $D(r, t)=[\varphi(r)]^{-1} \varphi(t)$ for some strictly decreasing real valued function, $\varphi:[0, \infty) \rightarrow$ $(0,1]$ with $\varphi(0)=1$ (hence, $\varphi$ is a generating function).

(ii) If $D$ is a continuous discount function, then $\varphi$ is onto (hence, $\varphi$ is a continuous generating function).

(iii) $D(0, t)=\varphi(t)$.

Proposition 5 justifies the following definition.

Definition 5 (Additive extensions): Let $\varphi$ be a generating function. Let $D(r, t)=$ $[\varphi(r)]^{-1} \varphi(t)$. Then:

(i) We call $\varphi$ the generating function of the additive discount function, $D$.

(ii) We call $D$ the additive extension of $\varphi$.

(iii) We also refer to $D(r, t)$ as the additive extension of $D(0, t)$.

Proposition 6 : The discount functions (2.12), (2.13) and (2.14) are additive. In each case, $D(r, t)$ is the additive extension of $D(0, t)$ and $\varphi(t)=D(0, t)$ is the generating function for $D(r, t)$. However, (2.15) is not additive. (2.16) is additive if, and only if, $Q[w(t)-w(r)]=Q[w(t)]-Q[w(r)]$, in which case $D(r, t)=e^{Q[w(r)]-Q[w(t)]}$ is the additive extension of $D(0, t)=e^{-Q[w(t)]}$ and $e^{-Q[w(t)]}$ is the generating function.

Proposition 7 (Invariance to the choice of reference time): Assume that one of the following holds.

(i) $D_{+}(r, t)=D_{-}(r, t)=D(r, t)$ and $D(r, t)$ is additive.

(ii) $D_{+}(r, t)$ is additive and all outcomes are in the domain of gains

(iii) $D_{-}(r, t)$ is additive and all outcomes are in the domain of losses.

Then

$$
\begin{aligned}
& \left(\left(x_{1}, s_{1}\right),\left(x_{2}, s_{2}\right), \ldots,\left(x_{m}, s_{m}\right)\right) \preceq_{w_{0}, r}\left(\left(y_{1}, t_{1}\right),\left(y_{2}, t_{2}\right), \ldots,\left(y_{n}, t_{n}\right)\right) \\
\Leftrightarrow & \left(\left(x_{1}, s_{1}\right),\left(x_{2}, s_{2}\right), \ldots,\left(x_{m}, s_{m}\right)\right) \preceq_{w_{0}, 0}\left(\left(y_{1}, t_{1}\right),\left(y_{2}, t_{2}\right), \ldots,\left(y_{n}, t_{n}\right)\right) .
\end{aligned}
$$

Thus, if we have the same additive discount function for gains and losses (as is the case with LP) then the choice of the reference time, $r$, back to which all utilities are discounted, is irrelevant. Discounting back to time $r$ is equivalent to discounting back to time 0 . Hence, the same result holds, even if we have different additive discount functions for gains and losses, provided all outcomes are in the domain of gains or all outcomes are in the domain of losses. However, if our discount function is not additive, or if the discount function for gains is different from the discount function for losses and we have a mixture of gains and losses, then the optimal choice of the decision maker may depend on the reference point for time. 


\subsection{Impatience}

The following concepts are also useful.

Definition 6 (Impatience): A discount function, $D(r, s)$, exhibits ${ }^{13}$

declining impatience if $D(r, s)<D(r+t, s+t)$, for $t>0$ and $r<s$, constant impatience if $D(r, s)=D(r+t, s+t)$, for $t \geq 0$ and $r \leq s$,

increasing impatience if $D(r, s)>D(r+t, s+t)$, for $t>0$ and $r<s$.

Proposition 8 : Let $D(r, t)$ be the $R S$-discount function (2.15), then:

(a) If $0<\rho \leq 1$, then $D$ is subadditive.

(b) If $\rho>1$, then $D$ is neither subadditive, additive nor superadditive.

(c) (i) If $0<\tau<1$, then $D$ exhibits declining impatience.

(ii) If $\tau=1$, then $D$ exhibits constant impatience.

(iii) If $\tau>1$, then $D$ exhibits increasing impatience.

Scholten and Read (2006a), bottom of p1425, state: $\alpha>0$ implies subadditivity (incorrect), $\rho>1$ implies superadditivity (incorrect) and $0<\tau<1$ implies declining impatience (correct but incomplete). Proposition 8 clarifies these points.

In the light of Proposition 8, we can now see the interpretation of the parameters $\tau$ and $\rho$ in the RS-discount function (2.15). $\tau$ controls impatience, independently of the values of the other parameters $\alpha, \beta$ and $\rho$ : $0<\tau<1$, gives declining impatience, $\tau=1$ gives constant impatience and $\tau>1$ gives increasing impatience. If $0<\rho \leq 1$, then we get subadditivity, irrespective of the values of the other parameters $\alpha, \beta$ and $\tau$. However, if $\rho>1$, then (2.15) can be neither subadditive, additive nor superadditive (depending on the particular values of $r, s$ and $t$, we may have $D(r, s)<D(r+t, s+t), D(r, s)=$ $D(r+t, s+t)$ or $D(r, s)>D(r+t, s+t))$.

\subsection{Intransitive preferences: Real or apparent?}

Consider the following hypothetical situation. A decision maker prefers a payoff of 1 now to a payoff of 2 next period, i.e., (2, next period) $\prec(1$, now). The decision maker also prefers a payoff of 2 next period to a payoff of 3 two periods from now, i.e., (3, 2 two periods from now) $\prec(2$, next period). Finally, the same decision maker prefers a payoff of 3 two periods from now to a payoff of 1 now, i.e., (1, now $) \prec(3,2$ two periods from now). Schematically:

$$
(1, \text { now }) \prec(3,2 \text { two periods from now }) \prec(2 \text {, next period }) \prec(1 \text {, now }) \text {. }
$$

\footnotetext{
${ }^{13}$ Some authors use 'present bias' for what we call 'declining impatience'. But other authors use 'present bias' to mean that the discount function, $D(s, t)$ is declining in $t$. So we prefer 'declining impatience' to avoid confusion. It is common to use 'stationarity' for what we call 'constant impatience'. We prefer the latter, to be in conformity with 'declining impatience' and 'increasing impatience'.
} 
Ok and Masatlioglu (2007, p215) use a similar example to motivate their intransitive theory of relative discounting.

Alternatively, we may view (2.24) as due to a framing effect resulting in a shift in the reference point for time. Assume that the choice of reference time in each pairwise comparison is the sooner of the two dates, in conformity with Assumption A0, subsection 2.4. Then (2.24) can be formalized as follows.

$$
V_{0}(1,0)<V_{0}(3,2), V_{1}(3,2)<V_{1}(2,1), V_{0}(2,1)<V_{0}(1,0) .
$$

Thus, the decision maker prefers a payoff of 1 now to a payoff of 2 next period, both discounted back to the present. The decision maker also prefers a payoff of 2 next period to a payoff of 3 the following period, both discounted back to next period. Finally, the decision maker prefers a payoff of 3 in two periods from now to a payoff of 1 now, both discounted back to the present. If this view is accepted, then the apparent intransitivity in (2.24) arises from conflating $V_{0}(3,2)$ with $V_{1}(3,2)$ and $V_{1}(2,1)$ with $V_{0}(2,1)$. The following example shows that (2.25) is consistent with a reference-time theory of intertemporal choice.

Example 2 : Take the reference point for wealth be the current level of wealth, so each payoff is regarded as a gain to current wealth. Take the value function to be ${ }^{14}$

$$
v(x)=x^{\frac{1}{2}}(1+x)^{\frac{1}{2}}, x \geq 0 .
$$

Thus (working to five significant figures),

$$
v(1)=1.4142, v(2)=2.4495 \text { and } v(3)=3.4641 \text {. }
$$

As our discount function we take the Read-Scholten discount function (2.15) with $\alpha=$ $\beta=1$ and $\rho=\tau=\frac{1}{2}$ :

$$
D(r, t)=\left(1+\left(t^{\frac{1}{2}}-r^{\frac{1}{2}}\right)^{\frac{1}{2}}\right)^{-1} .
$$

Thus,

$$
D(0,1)=\frac{1}{2}, D(1,2)=0.60842 \text { and } D(0,2)=0.45679 .
$$

From (2.27) and (2.29) we get

$$
\begin{aligned}
& V_{0}(1,0)=v(1) D(0,0)=1.4142, \\
& V_{0}(3,2)=v(3) D(0,2)=1.5824, \\
& V_{1}(3,2)=v(3) D(1,2)=2.1076,
\end{aligned}
$$

\footnotetext{
${ }^{14}$ The reasons for this choice will become clear in sections 3.1 and 3.2 , below. Many other choices are possible.
} 


$$
\begin{aligned}
& V_{1}(2,1)=v(2) D(1,1)=2.4495 \\
& V_{0}(2,1)=v(2) D(0,1)=1.2248
\end{aligned}
$$

From (2.30) to (2.34), we get

$$
V_{0}(1,0)<V_{0}(3,2), V_{1}(3,2)<V_{1}(2,1), V_{0}(2,1)<V_{0}(1,0)
$$

confirming (2.25).

A consequence of Proposition 7 is that no additive discount function (e.g., exponential (2.12), PPL (2.13) or LP (2.14)) can explain (apparently) intransitive choices as exhibited in (2.24). The reason is that, under the conditions of that proposition, all utilities can be discounted back to time zero and, hence, can be compared and ordered.

\subsection{The common difference effect: Declining impatience or subadditivity?}

Let us reconsider the common difference effect, using Thaler's apples example (anomaly 3 in the list of subsection 1.1). A decision maker prefers one apple today to two apples tomorrow, so that

$$
v(1)>v(2) D(0,1) .
$$

However, the decision maker, today, prefers to receive two apples in 51 days' time to receiving one apple in 50 days' time, so that

$$
v(1) D(0,50)<v(2) D(0,51) \text {. }
$$

From (2.36) and (2.37) we get

$$
D(0,50) D(0,1)<D(0,51)
$$

If we assume additivity (as did LP), so that $D(0,51)=D(0,50) D(50,51$ ), we get, from (2.38), $D(0,1)<D(50,51)$. So the decision maker exhibits declining impatience. However, and as Read (2001) pointed out, subadditivity could be an alternative explanation. To see this, assume constant impatience, so that $D(0,1)=D(50,51)$. Then $(2.38)$ gives $D(0,50) D(50,51)<D(0,51)$. Thus, the common difference effect is consistent with constant impatience if the discount function is sufficiently subadditive.

Example 3 : (Thaler's apples example)

Take the reference point to be 'no apples' and take the value function to be $e^{15}$

$$
v(x)=x^{\frac{1}{2}}(1+x)^{\frac{1}{2}}, x \geq 0 .
$$

\footnotetext{
${ }^{15}$ The reasons for this choice will become clear in sections 3.1 and 3.2, below. Many other choices will also do.
} 
Thus (working to five significant figures),

$$
v(1)=1.4142 \text { and } v(2)=2.4495 .
$$

We compare the resolution of the 'common difference effect' anomaly under the LPdiscount function (2.14) and the RS-discount function (2.15). To simplify as much as possible, choose the parameters: $\alpha=\beta=\tau=\rho=1$. We shall use these parameters in other examples too. We tabulate the relevant magnitudes below:

\begin{tabular}{|l|l|l|}
\hline & $L P: D(s, t)=(1+s)(1+t)^{-1}$ & RS: $D(s, t)=(1+t-s)^{-1}$ \\
\hline$D(0,1)$ & $1 / 2$ & $1 / 2$ \\
\hline$D(0,50)$ & $1 / 51$ & $1 / 51$ \\
\hline$D(0,51)$ & $1 / 52$ & $1 / 52$ \\
\hline$D(50,51)$ & $51 / 52$ & $1 / 2$ \\
\hline$D(0,50) D(50,51)$ & $1 / 52$ & $1 / 102$ \\
\hline
\end{tabular}

Recall that the decision maker prefers one apple today to two apples tomorrow if, and only if,

$$
v(1)>v(2) D(0,1) \text {. }
$$

On the other hand, the decision maker, today, prefers to receive two apples in 51 days' time to receiving one apple in 50 days' time if, and only if,

$$
v(1) D(0,50)<v(2) D(0,51) \text {. }
$$

Substituting from the above table and (2.40) into (2.41) and (2.42) gives, respectively, ${ }^{16}$

$$
\begin{gathered}
v(1)=1.4>1.2=v(2) D(0,1), \\
v(1) D(0,50)=0.028<2.4=v(2) D(0,51),
\end{gathered}
$$

for both the LP-discount function and the RS-discount function. This illustrates that both approaches can explain the common difference effect. However, they explain in very different ways. Comparing rows one and four of the table, we see that the LP-discount function exhibits declining impatience, $D(0,1)=\frac{1}{2}<\frac{51}{52}=D(50,51)$, while the RSdiscount function exhibits constant impatience, $D(0,1)=\frac{1}{2}=D(50,51)$. On the other hand, comparing rows three and five of the table, we see that the LP-discount function is additive, $D(0,51)=\frac{1}{52}=D(0,50) D(50,51)$, while the RS-discount function subadditive, $D(0,51)=\frac{1}{52}>\frac{1}{102}=D(0,50) D(50,51)$. Thus, the LP-discount function explains the common difference effect as exclusively due to declining impatience, while the RS-discount function explains this effect as due (in this example, exclusively) to subadditivity. More

\footnotetext{
${ }^{16}$ In the presentation of the results, we write down the first two significant figures, which is entirely adequate.
} 
generally, and provided $0<\rho \leq 1$, the RS-discount function can combine subadditivity with declining impatience $(0<\tau<1)$, constant impatience $(\tau=1)$ or increasing impatience $(\tau>1)$.

Of course, and as Read (2001) pointed out, the common difference effect could be due to both declining impatience and subadditivity. Read (2001), conducted a series of experiments that tested for the common difference effect and could also discriminate between subadditivity and declining impatience. He found support for the common difference effect and for subadditivity but rejected declining impatience in favour of constant impatience. Read (2001) also discusses the psychological foundation for subadditivity.

\subsection{Representation theorems}

Suppose that $x$ received at time 0 is equivalent to $y$ received at time $t$ (when both are discounted back to time 0 ), so that $v(x)=v(y) D(0, t)$. Suppose that the receipt of $x$ is delayed to time $s$. We ask, at what time, $T$, will $y$ received at time $T$ be equivalent to $x$ received at time $s$ (when both are discounted back to time 0)? Or, for what time, $T$, will the following hold: $v(x) D(0, s)=v(y) D(0, T)$ ? For the exponential discount function (2.12) the answer is clear: $T=s+t$. More generally, does such a $T$ exist? Is it unique? Does it depend on $x, y$ as well as $s, t$ ? What are its properties? These questions are answered by Propositions 9 and 10, below. But first, a definition.

Definition 7 (Delay functions): Let $D$ be a discount function. Suppose the function, $\Psi$, has the property $D(0, s) D(0, t)=D(0, \Psi(s, t)), s \geq 0, t \geq 0$. Then we call $\Psi$ a delay function corresponding to the discount function, $D$. We also say that the discount function, $D$, exhibits $\Psi$-delay.

Proposition 9 (Properties of a delay function): Let $D$ be a discount function and $\Psi$ a corresponding delay function. Then $\Psi$ has the following properties:

(a) $\Psi$ is unique,

(b) $\Psi(s, t)$ is strictly increasing in each of $s$ and $t$,

(c) $\Psi(s, t)=\Psi(t, s)$,

(d) $\Psi(0, t)=\Psi(t, 0)=t$,

(e) $v(x)=v(y) D(0, t)$ if, and only if, $v(x) D(0, s)=v(y) D(0, \Psi(s, t))$.

Suppose that $x$ received at time 0 is equivalent to $y$ received at time $t$ (when both are discounted back to time 0), so that $v(x)=v(y) D(0, t)$. Suppose that the receipt of $x$ is delayed to time $s$. Then, according to Proposition $9(\mathrm{e})$, the delay function, $\Psi(s, t)$, if it exists, gives the time to which the receipt of $y$ has to be deferred, so as to retain equivalence to $x$ (when both are discounted back to time 0 ). Therefore, we called $\Psi$ a delay function. 
Proposition 10 (Existence of a delay function): A continuous discount function has a unique delay function.

We now introduce our fourth defined function (the others were: the discount function, the generating function and the delay function).

Definition 8 (The seed function): Let $\psi:[0, \infty) \rightarrow[0, \infty)$ be strictly increasing, with $\psi(0)=0$. We call $\psi$ a seed function. If, in addition, $\psi$ is onto, we call $\psi$ a continuous seed function.

A 'continuous seed function' is continuous. The proof is similar to that of Proposition 1 and, therefore, will be omitted.

The following definition gives a useful representation for discount functions.

Definition 9 (Representations): Let $\alpha>0$ and $\beta>0$. We call $D(0, t)=[1+\alpha \psi(t)]^{-\frac{\beta}{\alpha}}$ an $(\alpha, \beta)$-representation of the discount function $D(r, t)$.

Proposition 11, below, establishes the existence of $(\alpha, \beta)$-representations for continuous discount functions and shows their connection to delay functions.

Proposition 11 (Representation Theorem 1): Let $D$ be a continuous discount function. Let $\Psi$ be the corresponding delay function. Then, for each $\alpha>0$ and each $\beta>0, D$ has the unique $(\alpha, \beta)$-representation $D(0, t)=[1+\alpha \psi(t)]^{-\frac{\beta}{\alpha}}$. Moreover, $\psi$ has the properties: (a) $\psi:[0, \infty) \stackrel{\text { onto }}{\longrightarrow}[0, \infty)$ is strictly increasing (hence $\psi(0)=0$ and $\psi$ is a continuous seed function).

(b) $\psi^{-1}$ exists and $\psi^{-1}:[0, \infty) \stackrel{\text { onto }}{\longrightarrow}[0, \infty)$ is strictly increasing with $\psi^{-1}(0)=0$.

(c) $\Psi(s, t)=\psi^{-1}[\psi(s)+\psi(t)+\alpha \psi(s) \psi(t)]$.

From Proposition 11, we see that if $\Psi$ is to be the delay function of some continuous discount function, then it must take the form given in part (c) of that proposition. In the light of this, when considering possible delay functions, we can restrict ourselves, without loss of generality, to the class of functions of the form $\Psi(s, t)=\psi^{-1}[\psi(s)+\psi(t)+\alpha \psi(s) \psi(t)]$, where $\psi$ is as in part (a), i.e., a continuous seed function.

The following proposition is a generalization of LP's derivation of their generalized hyperbolic discount function.

Proposition 12 (Representation Theorem 2): Let $\psi:[0, \infty) \stackrel{\text { onto }}{\rightarrow}[0, \infty)$ be strictly increasing, $\alpha>0$ and $\Psi(s, t)=\psi^{-1}[\psi(s)+\psi(t)+\alpha \psi(s) \psi(t)]$. Let $D$ be a continuous discount function with delay function, $\Psi$. Then, for some $\beta>0, D(0, t)=[1+\alpha \psi(t)]^{-\frac{\beta}{\alpha}}$. 
According to Propositions 9(a) and 10, a continuous discount function determines a unique delay function. Hence, we can partition the set of all continuous discount functions into equivalence classes, two continuous discount functions being in the same equivalence class if, and only if, they have the same delay function. Many different discount functions could have the same delay function. In fact, according to Representation Theorem 1 (Proposition 11), all (the different) continuous discount functions, $D$, for which $D(0, t)=$ $[1+\alpha \psi(t)]^{-\frac{\beta}{\alpha}}$ (fixed $\alpha$ and $\psi$, different $\beta$ 's) have the same delay function and, hence, lie in the same equivalence class. But does an equivalence class contain other continuous discount functions? Representation Theorem 2 (Proposition 12) gives the answer 'no': Consider an arbitrary equivalence class. Choose some member of that class. Let it have the $(\alpha, \beta)$-representation $D(0, t)=[1+\alpha \psi(t)]^{-\frac{\beta}{\alpha}}$. Then all members of its class can be obtained by varying $\beta$, keeping $\alpha$ and $\psi$ fixed.

\subsection{Extension theorems}

Proposition 8(a) and (b) established that the RS-discount function (2.15) is not additive and, hence, cannot be obtained as an additive extension of a strictly decreasing function $\varphi:[0, \infty) \rightarrow(0,1], \varphi(0)=1$. We, therefore, need a more general way to extend such a strictly decreasing function to a discount function. This is what we turn to in this subsection. We start by introducing our fifth, and final, defined function.

Definition 10 (extension functions): Let $f: \Delta \rightarrow[0, \infty)$ satisfy:

(i) For each $r \in[0, \infty), f(r, t)$ is a strictly increasing function of $t \in[r, \infty)$ into $[0, \infty)$, with $f(r, r)=0$.

(ii) For each $t \in[0, \infty), f(r, t)$ is a strictly decreasing function of $r \in[0, t]$ into $[0, t]$, with $f(0, t)=t$.

Then we call $f$ an extension function. If, in (i), 'into' is replaced with 'onto', then we call $f$ a continuous extension function.

A 'continuous extension function', $f(r, t)$, is continuous in $t$. The proof is the same as that of Proposition 1 and, therefore, will be omitted.

Definition 11 ( $f$-extensions): Let $D$ be a discount function. Let $f: \Delta \rightarrow[0, \infty)$ satisfy $D(r, t)=D(0, f(r, t))$, then

(a) we call $f$ an extension function corresponding to $D$,

(b) we refer to $D(r, t)$ as an $f$-extension of $D(0, t)$, or just an $f$-extension.

Definition 10 defines extension functions independently of any discount function. By contrast, Definition 11 defines an extension function corresponding to a give discount function. Our terminology suggests that 'an extension function corresponding to a given 
discount function' is, in fact, 'an extension function'. That this is indeed the case, is established in the following proposition.

Proposition 13 (Extension Theorem 1): Let $D$ be a discount function. Let $f$ be a corresponding extension function in the sense of Definition 11(a). Then:

(a) $f$ is unique.

(b) $f$ is an extension function in the sense of Definition 10.

(c) $v(x)=v(y) D(r, t)$ if, and only if, $v(x)=v(y) D(0, f(r, t))$.

Suppose that $x$ received at time $r$ is equivalent to $y$ received at time $t, 0 \leq r \leq t$, time $r$ being the reference time; so that $v(x)=v(y) D(r, t)$. Suppose that the receipt of $x$ is brought forward to time 0 . We ask, at what time, $T$, will $y$ received at time $T$ be equivalent to $x$ received at time 0 , time 0 being the new reference time? Or, for what time, $T$, will the following hold: $v(x)=v(y) D(0, T)$ ? For the exponential discount function (2.12) the answer is clear: $T=t-r$. More generally, Proposition 13(c) gives the answer as $T=f(r, t)$.

Proposition 14 (Extension Theorem 2): Let the discount function, D, be continuous. Then there exists an extension function, $f$, corresponding to $D$ in the sense of Definition 11. Moreover, $f$ is unique and is a continuous extension function in the sense of Definition 10.

Proposition 15 (Extension Theorem 3): Let $\varphi$ be a generating function and $f$ an extension function. Then:

(a) $D(r, t)=\varphi(f(r, t))$ is a discount function.

(b) $f$ is the extension function corresponding to $D$ and $D(r, t)$ is the $f$-extension of $D(0, t)$.

(c) If $\varphi$ is a continuous generating function and $f$ a continuous extension function, then $D$ is a continuous discount function.

To summarize, given a generating function, $\varphi$, and an extension function, $f$, by Extension Theorem 3 (Proposition 15), we can construct a discount function $D$ so that $D(r, t)$ is the $f$-extension of $\varphi(t)=D(0, t)$. Extension Theorem 2 (Proposition 14) tells us that all continuous discount functions are obtainable in this way from continuous generating functions and continuous extension functions.

\subsection{Characterization theorems}

We can combine the representation and extension theorems of the previous two subsections to produce further useful results, which we now turn to. 
Proposition 16 (Characterization Theorem 1): $D$ is a continuous discount function if, and only if, $D(r, t)=[1+\alpha \psi(f(r, t))]^{-\frac{\beta}{\alpha}}, \alpha>0, \beta>0$, where $\psi$ is a continuous seed function and $f$ is a continuous extension function. Furthermore, $f$ is uniquely determined by $D$.

Proposition 17 (Characterization Theorem 2): A continuous discount function with the extension function, $f$, exhibits:

(a) declining impatience if, and only if, $f(r, s)>f(r+t, s+t)$, for $t>0$ and $r<s$,

(b) constant impatience if, and only if, $f(r, s)=f(r+t, s+t)$, for $t \geq 0$ and $r \leq s$,

(c) increasing impatience if, and only if, $f(r, s)<f(r+t, s+t)$, for $t>0$ and $r<s$,

Proposition 18 (Characterization Theorem 3): A continuous discount function, $D$, is additive if, and only if, $f(r, t)=\psi^{-1}\left(\frac{\psi(t)-\psi(r)}{1+\alpha \psi(r)}\right)$, in which case $D(r, t)=\left[\frac{1+\alpha \psi(t)}{1+\alpha \psi(r)}\right]^{-\frac{\beta}{\alpha}}$.

Proposition 19 : The following two tables give a seed function, $\psi$, the generating function, $\varphi$, the extension function, $f$, and the delay function, $\Psi$, of each of the discount functions $D(r, t)(2.12)$ to $(2.15)$.

\begin{tabular}{|l|l|l|l|l|}
\hline & $\psi(t)$ & $\varphi(t)$ & $f(r, t)$ & $\Psi(s, t)$ \\
\hline exponential & $\frac{e^{\alpha t}-1}{\alpha}$ & $e^{-\beta t}$ & $t-r$ & $s+t$ \\
\hline $\boldsymbol{R S}$ & $t$ & $(1+\alpha t)^{-\frac{\beta}{\alpha}}$ & $\frac{t-r}{1+\alpha r}$ & $s+t+\alpha s t$ \\
\hline generalized $\boldsymbol{R S}$ & $\frac{e^{\frac{\alpha}{\beta} Q[w(t)]}-1}{\alpha}$ & {$\left[1+\alpha t^{\tau \rho}\right]^{-\frac{\beta}{\alpha}}$} & $\left(t^{\tau}-r^{\tau}\right)^{\frac{1}{\tau}}$ & {$\left[s^{\tau \rho}+t^{\tau \rho}+\alpha(s t)^{\tau \rho}\right]^{\frac{1}{\tau \rho}}$} \\
\hline $\boldsymbol{P P L}(r=t=0)$ & 0 & 1 & $w^{-1}[w(t)-w(r)]$ & $w^{-1} Q^{-1}[Q(w(s))+Q(w(t))]$ \\
\hline $\boldsymbol{P P L}(0=r<t)$ & $\frac{e^{\frac{\alpha \delta}{\beta}+\alpha t}-1}{\alpha}$ & $e^{-(\delta+\beta t)}$ & $t$ & $s$ \\
\hline $\boldsymbol{P P L}(0<r \leq t)$ & $\frac{e^{\alpha t}-1}{\alpha}$ & $e^{-\beta t}$ & $\frac{\delta}{\beta}+s+t$ \\
\hline \hline & $D(r, t)$ & & $N A$ \\
\hline exponential & $e^{-\beta(t-r)}$ & & \\
\hline LP & $\left(\frac{1+\alpha t}{1+\alpha r}\right)^{-\frac{\beta}{\alpha}}$ & & \\
\hline $\boldsymbol{R S}$ & {$\left[1+\alpha\left(t^{\tau}-r^{\tau}\right)^{\rho}\right]^{-\frac{\beta}{\alpha}}$} \\
\hline generalized $\boldsymbol{R S}$ & $e^{-Q[w(t)-w(r)]}$ \\
\hline $\boldsymbol{P P L}(r=t=0)$ & 1 & \\
\hline $\boldsymbol{P P L}(0=r<t)$ & $e^{-(\delta+\beta t)}$ & \\
\hline PPL $(0<r \leq t)$ & $e^{-\beta(t-r)}$ & \\
\hline
\end{tabular}

Starting with a continuous seed function, $\psi$, an $\alpha>0$ and a $\beta>0$, we can 'grow' from them a unique generating function, $\varphi(t)=[1+\alpha \psi(t)]^{-\frac{\beta}{\alpha}}$ (which turns out to be continuous). Given this generating function and a continuous extension function, $f(r, t)$, we obtain a unique discount function $D(r, t)=\varphi(f(r, t))=[1+\alpha \psi(f(r, t))]^{-\frac{\beta}{\alpha}}$ (which also turns out to be continuous). This discount function determines a unique delay function, $\Psi(s, t)=\psi^{-1}[\psi(s)+\psi(t)+\alpha \psi(s) \psi(t)]$. 
Conversely, a continuous discount function, $D$, determines a unique (continuous) generating function, $\varphi(t)=D(0, t)$ and a unique (continuous) extension function, $f$, so that $D$ is the $f$-extension of $\varphi: D(r, t)=\varphi(f(r, t))$.

Although a continuous discount function, $D$, determines unique generating, extension and delay functions, $\varphi, f$ and $\Psi$, it does not determine unique $\alpha, \beta$ or $\psi$ in the representation $D(0, t)=[1+\alpha \psi(t)]^{-\frac{\beta}{\alpha}}$. For example, the LP-discount function $D(r, t)=$ $(1+\alpha r)^{\frac{\beta}{\alpha}}(1+\alpha t)^{-\frac{\beta}{\alpha}}, \alpha>0, \beta>0$, has, obviously, the representation $D(0, t)=(1+\alpha t)^{-\frac{\beta}{\alpha}}$ (with $\psi(t)=t$ ) and, hence, the delay function $\Psi(s, t)=s+t+\alpha s t$. But it also has many other representations: $D(0, t)=[1+a \psi(t)]^{-\frac{b}{a}}, \psi(t)=\frac{(1+\alpha t)^{\frac{\beta a}{\alpha b}}-1}{a}$, for all $a>0$ and all $b>0$. However, it can easily be check that $[1+a \psi(t)]^{-\frac{b}{a}}=(1+\alpha t)^{-\frac{\beta}{\alpha}}$ and $\psi^{-1}[\psi(s)+\psi(t)+a \psi(s) \psi(t)]=s+t+\alpha s t$. Since the delay function, $\Psi$, but not the seed function, $\psi$, is uniquely determined by $D$, it is better to say that $D$ exhibits $\Psi$ delay rather than $\psi$ delay.

\subsection{Assumptions and consequences}

LP introduce four assumptions, all with good experimental support (LP, II pp574-578). We adapt these assumption to allow for (i) discount functions that are not, necessarily, additive and (ii) discount functions that may be different for gains and losses. Under these latter two conditions, the reference point for time becomes important (Proposition 7). Let the discount functions for gains, $D_{+}$, and for losses, $D_{-}$, be given by:

$$
\begin{aligned}
& D_{+}(r, t)=\left[1+\alpha_{+} \psi_{+}\left(f_{+}(r, t)\right)\right]^{-\frac{\beta_{+}}{\alpha_{+}}}, \alpha_{+}>0, \beta_{+}>0, \\
& D_{-}(r, t)=\left[1+\alpha_{-} \psi_{-}\left(f_{-}(r, t)\right)\right]^{-\frac{\beta_{-}}{\alpha_{-}}}, \alpha_{-}>0, \beta_{-}>0,
\end{aligned}
$$

where $\psi_{+}$and $f_{+}$are, respectively, the seed and extension functions for gains. Analogously, $\psi_{-}$and $f_{-}$are, respectively, the seed and extension functions for losses. If $D_{+}$is a continuous discount function then it can always be represented in the form (2.45), where $\psi_{+}$is a continuous seed function and $f_{+}$is a continuous extension function. Moreover, $f_{+}$ is determined uniquely by $D_{+}$(Characterization Theorem 1 (Proposition 16)). Analogous statements hold for the discount function for losses, $D_{-}$. Furthermore, under the assumption of continuity, $D_{+}$and $D_{-}$will have unique delay functions, $\Psi_{+}$and $\Psi_{-}$, respectively (Propositions 9 and 10 and Representation Theorem 1 (Proposition 11)), and are given by:

$$
\begin{aligned}
& \Psi_{+}(s, t)=\psi_{+}^{-1}\left[\psi_{+}(s)+\psi_{+}(t)+\alpha_{+} \psi_{+}(s) \psi_{+}(t)\right] \\
& \Psi_{-}(s, t)=\psi_{-}^{-1}\left[\psi_{-}(s)+\psi_{-}(t)+\alpha_{-} \psi_{-}(s) \psi_{-}(t)\right] .
\end{aligned}
$$

Assumptions A1 to A4, below, correspond to anomalies 1 to 4, above (subsection 1.1). 
Thus, what is regarded as anomalous behavior from the neoclassical point of view is at the core of the RT theory.

Given discount functions, $D_{ \pm}(r, t)$, for gains $(+)$ and losses $(-)$ respectively, the assumption A1 to A4, below, place restrictions only on $D_{ \pm}(0, t)$, i.e., only on discounting from an arbitrary time, $t \geq 0$, back to time zero. Hence, to derive results for $D_{ \pm}(r, t)$, further assumptions are needed. In particular, for Proposition 24 we assume that $D_{ \pm}(r, t)$ is an additive extension of $D_{ \pm}(0, t)$, while for Proposition 25 we assume that $D_{ \pm}(r, t)$ is an $f$-extension of $D_{ \pm}(0, t)$ for $f_{ \pm}(r, t)=\left(t^{\tau_{ \pm}}-r^{\tau_{ \pm}}\right)^{\frac{1}{\tau_{ \pm}}}$.

A1 Gain-loss asymmetry. If $0<x<y$ and $v(x)=v(y) D_{+}(0, t)$, then $v(-x)>$ $v(-y) D_{-}(0, t)$.

A2 Magnitude effect. If $0<x<y, v(x)=v(y) D_{+}(0, t)$ and $a>1$, then $v(a x)<$ $v(a y) D_{+}(0, t)$. If $y<x<0, v(x)=v(y) D_{-}(0, t)$ and $a>1$, then $v(a x)>$ $v(a y) D_{-}(0, t)$.

A3 Common difference effect. If $0<x<y, v(x)=v(y) D_{+}(0, t)$ and $s>0$, then $v(x) D_{+}(0, s)<v(y) D_{+}(0, s+t)$. If $y<x<0, v(x)=v(y) D_{-}(0, t)$ and $s>0$, then $v(x) D_{-}(0, s)>v(y) D_{-}(0, s+t)$.

A4 Delay-speedup asymmetry. For $c>0, s>0$ and $t>0, V_{0}((0,0),(c, s),(-c, s+t))<$ $-V_{0}((0,0),(-c, s),(c, s+t)) \cdot{ }^{17}$

Definition 12 ( $\alpha$-subadditivity): Let $\alpha>0$. A function, $\psi$, is $\alpha$-subadditive if, for all $s$ and $t$ for which $\psi$ is defined and non-zero: $\psi(s+t)<\psi(s)+\psi(t)+\alpha \psi(s) \psi(t)$.

A function, $\psi$, is subadditive (in the standard sense) if, for all $s$ and $t$ for which $\psi$ is defined: $\psi(s+t) \leq \psi(s)+\psi(t)$. A function that is $\alpha$-subadditive, for some $\alpha>0$, need not be subadditive. However, a function is subadditive if, and only if, it is $\alpha$-subadditive for all $\alpha>0 .^{18}$

\footnotetext{
${ }^{17} \mathrm{~A} 4$ is to be understood as follows. In the LHS of the inequality, the reference stream of the decision maker is $0,0, c$ (for dates $0, s, s+t$ ) i.e. a reward is contractually promised at time $s+t>0$. The individual is then offered a choice to receive the reward early, at time $s$ (speedup). Given the assumption on reference time, in $\mathrm{A} 0$, the income stream, relative to reference wealth, $0, c,-c$, can be explained as follows. The individual was not expecting anything at times 0 and $s$ so relative to reference wealth, he gets $0-0, c-0$ at times 0 and $s$. Having received a reward of $c$ at time $s$, his reference wealth is $c$. Hence, at time $t+s$ his wealth relative to the reference wealth is $0-c=-c$. For the RHS of the inequality, the contractually promised income stream is $0, c, 0$ (for dates $0, s, s+t$ ). The individual is then told that the reward will now instead be available only at time $s+t$ (delay). Proceeding as before, the stream of income relative to the reference point is now $0,-c, c$.

${ }^{18}$ Similarly, a function, $\psi$, is additive (in the standard sense) if, for all $s$ and $t$ for which $\psi$ is defined: $\psi(s+t)=\psi(s)+\psi(t)$. Consider the exponential discount function, $D(r, t)=e^{-\beta(t-r)}, \beta>0$. Then $\ln D(0, t)$ is additive in this sense. And, of course, $D(r, t)$ is additive in the sense of Definition 3. Also note that $\alpha$-subaddivity, as in Definition 12, neither implies nor is implied by subadditivity of the discount function, as in Definition 3.
} 
Proposition 20 (Representation Theorem 3): Let $D$ be a continuous discount function. Let $\Psi$ be the corresponding delay function. Let $\alpha>0$ and $\beta>0$. Let $D(0, t)=$ $[1+\alpha \psi(t)]^{-\frac{\beta}{\alpha}}$ be an $(\alpha, \beta)$-representation of $D$. Then the following are equivalent:

(a) $\psi$ is $\alpha$-subadditive.

(b) If $s>0$ and $t>0$, then $\Psi(s, t)>s+t$.

(c) If $s>0$ and $t>0$, then $D(0, s) D(0, t)<D(0, s+t)$.

Proposition 21 (Characterization Theorem 4): Let $D$ be a continuous discount function. Then preferences exhibit the common difference effect for gains if, and only if, the seed function for gains, $\psi_{+}$, is $\alpha_{+}$-subadditive. ${ }^{19}$ Similarly, preferences exhibit the common difference effect for losses if, and only if, the seed function for losses, $\psi_{-}$, is $\alpha_{-}$-subadditive.

Definition 13 ( $\gamma$-delay): Preferences exhibit $\gamma$-delay, if the delay functions for gains (2.47) and losses (2.48) are:

$$
\begin{aligned}
& \Psi_{+}(s, t)=\left(s^{\gamma_{+}}+t^{\gamma_{+}}+\alpha_{+} s^{\gamma_{+}} t^{\gamma_{+}}\right)^{\frac{1}{\gamma_{+}}}, \alpha_{+}>0,0<\gamma_{+} \leq 1 \\
& \Psi_{-}(s, t)=\left(s^{\gamma_{-}}+t^{\gamma_{-}}+\alpha_{-} s^{\gamma_{-}} t^{\gamma_{-}}\right)^{\frac{1}{\gamma_{-}}}, \alpha_{-}>0,0<\gamma_{-} \leq 1
\end{aligned}
$$

In particular, if $\gamma_{+}=\gamma_{-}=1$, we say that preferences exhibit linear delay.

A delay function, if it exists, is unique (Proposition 9) and it always exists for a continuous discount function (Proposition 10). Hence, Definition 13 is a sound definition. However, it should be remembered that $\gamma$-delay is a property of the delay function, $\Psi$, not of the seed function, $\psi$ (see discussion at end of subsection 2.12).

Proposition 22 : If preferences exhibit $\gamma$-delay, then they also exhibit the common difference effect.

Proposition 23 : If preferences, with continuous discount functions for gains and losses, $D_{+}$and $D_{-}$, respectively, exhibit $\gamma$-delay, then, necessarily, $D_{+}(0, t)=\left(1+\alpha_{+} t^{\gamma_{+}}\right)^{-\frac{\beta_{+}}{\alpha_{+}}}$ and $D_{-}(0, t)=\left(1+\alpha_{-} t^{\gamma_{-}}\right)^{-\frac{\beta_{-}}{\alpha_{-}}}$, where $\alpha_{+}, \alpha_{-}, \beta_{+}, \beta_{-}$are all positive.

Proposition 24 (generalization of LP): If preferences, with additive continuous discount functions for gains and losses, $D_{+}$and $D_{-}$, respectively, exhibit $\gamma$-delay, then, necessarily, $D_{+}(r, t)=\left(\frac{1+\alpha_{+} t^{\gamma+}}{1+\alpha_{+} r^{\gamma+}}\right)^{-\frac{\beta_{+}}{\alpha_{+}}}$and $D_{-}(r, t)=\left(\frac{1+\alpha_{-} t_{-}}{1+\alpha_{-} \gamma_{-}}\right)^{-\frac{\beta_{-}}{\alpha_{-}}}$, where $\alpha_{+}, \alpha_{-}, \beta_{+}, \beta_{-}$are all positive.

In particular, $\alpha_{+}=\alpha_{-}, \beta_{+}=\beta_{-}$and $\gamma_{+}=\gamma_{-}=1$ give the LP-discount function (2.14).

\footnotetext{
${ }^{19}$ We noted earlier that the common difference effect can be explained by a combination of declining impatience and subadditivity of the discount function. However, $\alpha_{+}$-subadditivity is not to be confused with subadditivity of the discount function.
} 
Proposition 25 (RS-discount functions): Let $\tau_{+}>0$ and $\tau_{-}>0$. Let $\rho_{+}=\frac{\gamma_{+}}{\tau_{+}}$and $\rho_{-}=\frac{\gamma_{-}}{\tau_{-}}$. Let $f_{+}(r, t)=\left(t^{\tau_{+}}-r^{\tau_{+}}\right)^{\frac{1}{\tau_{+}}}$and $f_{-}(r, t)=\left(t^{\tau_{-}}-r^{\tau_{-}}\right)^{\frac{1}{\tau_{-}}}$. Let preferences with continuous discount function $D_{+}$(gains) and $D_{-}$(losses) exhibit $\gamma$-delay. Let the extension function of $D_{+}$be $f_{+}$and that of $D_{-}$be $f_{-}$. Then $D_{+}$and $D_{-}$are the RSdiscount functions:

$D_{+}(r, t)=\left[1+\alpha_{+}\left(t^{\tau_{+}}-r^{\tau_{+}}\right)^{\rho_{+}}\right]^{-\frac{\beta_{+}}{\alpha_{+}}}$,

$D_{-}(r, t)=\left[1+\alpha_{-}\left(t^{\tau_{-}}-r^{\tau_{-}}\right)^{\rho_{-}}\right]^{-\frac{\beta_{-}}{\alpha_{-}}}$,

where $\alpha_{+}, \alpha_{-}, \beta_{+}, \beta_{-}$are all positive. ${ }^{20}$

Proposition 26 (LP): Suppose that the continuous discount function, $D_{ \pm}$, satisfies $D_{-}=$ $D_{+}$. Then $A 1$ implies the following:

(a) Losses are discounted less heavily than gains in the following sense: $0<x<y \Rightarrow$ $\frac{v(x)}{v(y)}>\frac{v(-x)}{v(-y)}$.

(b) The value function is more elastic for losses than for gains: $x>0 \Rightarrow \epsilon_{v}(-x)>\epsilon_{v}(x)$.

Proposition $27(L P)$ : For a continuous discount function, A2 implies that the value function is

(a) subproportional: $(0<x<y$ or $y<x<0) \Rightarrow \frac{v(x)}{v(y)}>\frac{v(a x)}{v(a y)}$, for $a>1$,

(b) more elastic for outcomes of larger absolute magnitude: $(0<x<y$ or $y<x<0) \Rightarrow$ $\epsilon_{v}(x)<\epsilon_{v}(y) .^{21}$

Intuitively, increasing elasticity of the value function implies greater sensitivity of $v$ to increases in $x$. This in turn increases the weight of larger outcomes $\left(D_{ \pm}(r, t) v\left(x_{t}\right)\right)$ in intertemporal plans. A similar intuition applies to the result in Proposition 26(b).

We now add two standard assumption from prospect theory. The first is that the value function is strictly concave for gains and strictly convex for losses (Kahneman and Tversky, 1979):

A5 Declining sensitivity. For $x>0, v^{\prime \prime}(x)<0$ (strict concavity for gains). For $x<0$, $v^{\prime \prime}(x)>0$ (strict convexity for losses).

Combining A5 with Proposition 27 we get:

Proposition 28:A2 and A5 imply that $0<\epsilon_{v}<1$.

\footnotetext{
${ }^{20}$ Strictly speaking, what we call as the RS-discount function is a slight generalization of the discount function in Scholten and Read (2006a). They do not have a reference point for time/wealth, nor do they have separate discounting for gains and losses.

${ }^{21}$ This proposition is stated incorrectly in al-Nowaihi and Dhami (2006a).
} 
The second assumption that we add from prospect theory is constant loss aversion. While this is not core to prospect theory, it is very useful and has good empirical support (Tversky and Kahneman, 1991 and 1992):

A6 Constant loss aversion. $v(-x)=-\lambda v(x), \lambda>1$, for $x>0$,

With the aid of these two extra assumptions, we get the following two theorems:

Proposition 29 : From A1 and A6 it follows that, for a continuous discount function:

(a) $t>0 \Rightarrow D_{+}(0, t)<D_{-}(0, t)$,

(b) $0<x<y \Rightarrow \frac{v(x)}{v(y)}=\frac{v(-x)}{v(-y)}$,

(c) $x>0 \Rightarrow \epsilon_{v}(-x)=\epsilon_{v}(x)$.

Proposition 30 : Assumption A4 (delay-speedup asymmetry) follows from the other assumptions, in particular A0 (reference time), A1 (gain-loss asymmetry) and A6 (constant loss aversion).

As mentioned above, LP assume that $D_{+}=D_{-}$. While this is consistent with their theory, it does not follow from it. Assuming that $D_{+}=D_{-}$is obviously attractive. However, it implies (Proposition 26) that gain-loss asymmetry (A1) can only be satisfied if $\epsilon_{v}(-x)>\epsilon_{v}(x)$, for $x>0$. In the light of Proposition 29, this would exclude value functions exhibiting constant loss aversion. While constant loss aversion is not core to prospect theory, this auxiliary assumption considerably simplifies application of the theory and is consistent with the evidence (Tversky and Kahneman, 1991, 1992).

Propositions 26 and 27 are due to LP. Proposition 24 extends LP from linear delay to $\gamma$-delay.

\section{Simple increasing elasticity value functions (SIE)}

A natural question that arises is 'Is the RT theory developed in section 2 consistent?' A related question is 'Is there a tractable functional form for the value function which can be combined with RT theory to produce a useful model?' We address these questions in this section. In subsection 3.2, below, we answer the second question in the affirmative. We call the value function developed there a simple increasing elasticity (SIE) value function. Our affirmative answer to the second question also provides an affirmative answer to the first question. But first, in subsection 3.1, immediately below, we show that none of several popular families of functions is compatible with RT theory or, indeed, any theory (e.g., LP) that attempts to explain the magnitude effect on the basis of increasing elasticity of the value function. 


\subsection{Incompatibility of HARA value functions with the reference-time theory}

We consider several popular classes of value functions including CARA (constant absolute risk aversion), CRRA (constant relative risk aversion), HARA (hyperbolic absolute risk aversion), logarithmic and quadratic. Proposition 31, below, shows that each member of this family exhibits constant or declining elasticity, contradicting LP's Proposition 27, which holds in the RT theory. Hence, none of these families is compatible with the RT theory (and, hence, none is compatible with the LP theory either). First, we give the definitions and main properties of this family of functions, followed by the main result of this subsection: Proposition 31.

Notation: We use the notation, $\rho_{A}$ and $\rho_{R}$ respectively, for the coefficients of absolute risk aversion and relative risk aversion. So for a utility function $v(x), \rho_{A}=-\frac{v^{\prime \prime}(x)}{v^{\prime}(x)}$ and $\rho_{R}=-\frac{x v^{\prime \prime}(x)}{v^{\prime}(x)}$.

\subsubsection{Constant relative risk aversion functions (CRRA)}

$$
\begin{aligned}
v(x) & =\frac{x^{1-\gamma}}{1-\gamma}, 0<\gamma<1, \\
v^{\prime}(x) & =x^{-\gamma}>0 ; v^{\prime \prime}(x)=-\gamma x^{-\gamma-1}<0, \\
\rho_{R} & =-\frac{x v^{\prime \prime}(x)}{v^{\prime}(x)}=\gamma, \\
\epsilon_{v}(x) & =\frac{x v^{\prime}(x)}{v(x)}=1-\gamma .
\end{aligned}
$$

The general restriction is that $\gamma \neq 1$. However, we need the stronger restriction, $0<\gamma<1$, in order to satisfy Proposition 28. It is clear, from the last line of (3.1), that members of the CRRA class of functions violate Proposition 27 and, hence, are not compatible with RT theory.

\subsubsection{Hyperbolic absolute risk aversion functions (HARA)}

$$
\begin{aligned}
v(x) & =\frac{\gamma}{1-\gamma}\left[\left(\mu+\frac{\theta x}{\gamma}\right)^{1-\gamma}-\mu^{1-\gamma}\right], \theta>0, \mu>0,0<\gamma<1, x \geq 0, \\
v^{\prime}(x) & =\theta\left(\mu+\frac{\theta x}{\gamma}\right)^{-\gamma}>0 ; v^{\prime \prime}(x)=-\theta^{2}\left(\mu+\frac{\theta x}{\gamma}\right)^{-\gamma-1}<0, \\
\epsilon_{v}(x) & =\frac{x v^{\prime}(x)}{v(x)}=\frac{(1-\gamma) \frac{\theta x}{\gamma}\left(\mu+\frac{\theta x}{\gamma}\right)^{-\gamma}}{\left(\mu+\frac{\theta x}{\gamma}\right)^{1-\gamma}-\mu^{1-\gamma}}, \\
\rho_{A} & =-\frac{v^{\prime \prime}(x)}{v^{\prime}(x)}=\frac{\gamma \theta}{\gamma \mu+\theta x} .
\end{aligned}
$$


The general restrictions are $\theta>0,\left(\mu+\frac{\theta x}{\gamma}\right)^{1-\gamma}>0, \gamma \neq 1$. Since we allow $x \in[0, \infty)$, the restriction $\left(\mu+\frac{\theta x}{\gamma}\right)^{1-\gamma}>0$ implies that $\mu>0$ and $\gamma>0$. We then also need $\gamma<1$ in order to satisfy Corollary 28.

Note that, traditionally, the HARA class is defined by $v(x)=\frac{\gamma}{1-\gamma}\left(\mu+\frac{\theta x}{\gamma}\right)^{1-\gamma}$, and that $\epsilon_{v}(x)=(1-\gamma)\left(1+\frac{\gamma \mu}{\theta x}\right)^{-1}$, which is increasing in $x$, as required by Proposition 27 . While an additive constant, of course, makes no difference in expected utility theory; its absence here would violate the assumption $v(0)=0$. However, including the constant $-\frac{\gamma}{1-\gamma} \mu^{1-\gamma}$, to make $v(0)=0$, results in $\epsilon_{v}(x)$ decreasing with $x$, as will be shown by Proposition 31, and, hence, violates Proposition 27.

The following three classes of functions are also regarded members of the HARA family.

\subsubsection{Constant absolute risk aversion functions (CARA)}

$$
\begin{aligned}
v(x) & =1-e^{-\theta x}, \theta>0, x \geq 0, \\
v^{\prime}(x) & =\theta e^{-\theta x}>0 ; v^{\prime \prime}(x)=-\theta^{2} e^{-\theta x}<0, \\
\rho_{A} & =-\frac{v^{\prime \prime}(x)}{v^{\prime}(x)}=\theta, \\
\epsilon_{v}(x) & =\frac{x v^{\prime}(x)}{v(x)}=\frac{\theta x}{e^{\theta x}-1}=\frac{1}{\sum_{n=1}^{\infty} \frac{(\theta x)^{n-1}}{n !}} .
\end{aligned}
$$

From the last line of (3.3), we see that $\epsilon_{v}(x)$ is decreasing with $x$. Hence, the CARA class is not compatible with the RT theory.

\subsubsection{Logarithmic functions}

$$
\begin{aligned}
v(x) & =\ln (1+\theta x), \theta>0, x \geq 0, \\
v^{\prime}(x) & =\theta(1+\theta x)^{-1}>0 ; v^{\prime \prime}(x)=-\theta^{2}(1+\theta x)^{-2}<0, \\
\epsilon_{v}(x) & =\frac{x v^{\prime}(x)}{v(x)}=\frac{\theta x}{(1+\theta x) \ln (1+\theta x)} .
\end{aligned}
$$

Proposition 31, below, establishes that $\epsilon_{v}(x)$ is decreasing with $x$. Hence this class is not compatible with the RT theory.

\subsubsection{Quadratic functions}

$$
\begin{aligned}
v(x) & =\frac{1}{2} \mu^{2}-\frac{1}{2}(\mu-\theta x)^{2}, \theta>0,0 \leq x<\frac{\mu}{\theta}, \\
v^{\prime}(x) & =\theta(\mu-\theta x)^{2}>0 ; v^{\prime \prime}(x)=-2 \theta^{2}(\mu-\theta x)<0 \\
\epsilon_{v}(x) & =\frac{x v^{\prime}(x)}{v(x)}=\frac{2 \theta x(\mu-\theta x)^{2}}{\mu^{2}-(\mu-\theta x)^{2}}
\end{aligned}
$$


Proposition 31, below, establishes that $\epsilon_{v}(x)$ is decreasing with $x$. Hence this class is also not compatible with the RT theory.

Proposition 31 : For members of the CRRA class of value functions (3.1), $\epsilon_{v}(x)$ is constant. For members of the HARA (3.2), CARA (3.3), logarithmic (3.4) and quadratic (3.5) classes of functions, $\epsilon_{v}(x)$ is declining. Hence none of these families is compatible with the RT theory.

\subsection{A value function compatible with the reference-time theory}

We do two things in this subsection. First, we provide a simple tractable functional form for the value function that is compatible with the RT theory. We call this a simple increasing elasticity (SIE) value function. Second, we provide a scheme for generating further such functions. This is important for two reasons. First, it provides a model for the RT theory and, therefore, establishes its internal consistency. Second, these functional forms may aid applications and further theoretical development.

The following method can be used to generate candidates for value functions compatible with the RT theory. Choose a function, $h(x)$, satisfying:

$$
0<h(x)<1, h^{\prime}(x)>0
$$

then solve the following differential equation for $v(x)$ :

$$
\frac{x}{v} \frac{d v}{d x}=h(x)
$$

This method only yields candidate value functions, which then have to be verified. For example, choose

$$
\begin{aligned}
h(x) & =\frac{a x}{b+x}+c, x \geq 0, \\
a & >0, b>0, c>0, a+c \leq 1 .
\end{aligned}
$$

Substituting from (3.8) into (3.7), separating variables, then integrating, gives:

$$
\begin{aligned}
\frac{x}{v} \frac{d v}{d x} & =\frac{a x}{b+x}+c \\
\int \frac{d v}{v} & =a \int \frac{d x}{b+x}+c \int \frac{d x}{x} \\
\ln v & =a \ln (b+x)+c \ln x+\ln K \\
v(x) & =K(b+x)^{a} x^{c} .
\end{aligned}
$$


Choosing $a=1-\gamma, b=\frac{\gamma \mu}{\theta}, c=\sigma$ and $K=\frac{\gamma}{1-\gamma}\left(\frac{\theta}{\gamma}\right)^{1-\gamma}$ produces a value function:

$$
v(x)=\frac{\gamma x^{\sigma}}{1-\gamma}\left(\mu+\frac{\theta}{\gamma} x\right)^{1-\gamma}, x \geq 0,
$$

The restrictions $a>0, b>0, c>0, a+c \leq 1$ give: $0<\sigma \leq \gamma<1$ and $\mu / \theta>0$. To ensure that $v^{\prime}>0$, take $\theta>0$. Hence $\mu>0$. For $x<0$, define $v(x)$ by $v(x)=-\lambda v(-x)$, where $\lambda>1$. Putting all these together gives the candidate value function

$$
\begin{aligned}
v(x) & =\frac{\gamma x^{\sigma}}{1-\gamma}\left(\mu+\frac{\theta}{\gamma} x\right)^{1-\gamma}, x \geq 0, \\
v(x) & =-\lambda v(-x) \\
& =-\lambda \frac{\gamma(-x)^{\sigma}}{1-\gamma}\left(\mu-\frac{\theta}{\gamma} x\right)^{1-\gamma}, x<0, \\
\mu & >0, \theta>0, \lambda>1,0<\sigma \leq \gamma<1,
\end{aligned}
$$

where $\lambda$ is the (constant) coefficient of loss aversion.

It may be interesting to note that (3.11) is a product of a CRRA function, $x^{\sigma}$, and a HARA function, $\frac{\gamma}{1-\gamma}\left(\mu+\frac{\theta}{\gamma} x\right)^{1-\gamma}$.

Proposition 32 : From (3.11) it follows that:

(a) $v:(-\infty, \infty) \rightarrow(-\infty, \infty), v(0)=0$, $v$ is continuous, $v$ is $C^{\infty}$ except at $x=0$.

(b) $v^{\prime}(x)=\frac{\sigma \gamma x^{\sigma-1}}{1-\gamma}\left(\mu+\frac{\theta}{\gamma} x\right)^{1-\gamma}+\theta x^{\sigma}\left(\mu+\frac{\theta}{\gamma} x\right)^{-\gamma}>0, x>0$,

(c) $v^{\prime \prime}(x)=\left(\mu+\frac{\theta}{\gamma} x\right)^{-\gamma-1}\left[-x^{\sigma-2}\left[\sigma \mu+(\sigma-\gamma) \frac{\theta x}{\gamma}\right]^{2}-\frac{x^{\sigma-2} \sigma(\gamma-\sigma)}{1-\gamma}\left(\mu+\frac{\theta}{\gamma} x\right)^{2}\right]<0, x>$ 0 ,

(d) $v^{\prime}(x)=\lambda v^{\prime}(-x)>0, x<0$,

(e) $v^{\prime \prime}(x)=-\lambda v^{\prime \prime}(-x)>0, x<0$,

(f) $\epsilon_{v}(x)=\frac{x}{v} \frac{d v}{d x}=\sigma+\frac{1-\gamma}{1+\frac{\gamma \mu}{\theta x}}>0, \epsilon_{v}^{\prime}(x)>0, x>0$,

(g) $\epsilon_{v}(x)=\frac{x}{v} \frac{d v}{d x}=\sigma+\frac{1-\gamma}{1-\frac{\gamma \mu}{\theta x}}>0, \epsilon_{v}^{\prime}(x)<0, x<0$,

(h) $\rho_{A}=-\frac{v^{\prime \prime}(x)}{v^{\prime}(x)}=\frac{(1-\gamma)\left[\sigma \mu+(\sigma-\gamma) \frac{\theta x}{\gamma}\right]^{2}+\sigma(\gamma-\sigma)\left(\mu+\frac{\theta}{\gamma} x\right)^{2}}{\sigma \gamma x\left(\mu+\frac{\theta}{\gamma} x\right)^{2}+\theta(1-\gamma) x^{2}\left(\mu+\frac{\theta}{\gamma} x\right)}>0, x>0$,

(i) $\rho_{R}=-\frac{x v^{\prime \prime}(x)}{v^{\prime}(x)}=\frac{(1-\gamma)\left[\sigma \mu+(\sigma-\gamma) \frac{\theta x}{\gamma}\right]^{2}+\sigma(\gamma-\sigma)\left(\mu+\frac{\theta}{\gamma} x\right)^{2}}{\sigma \gamma\left(\mu+\frac{\theta}{\gamma} x\right)^{2}+\theta(1-\gamma) x\left(\mu+\frac{\theta}{\gamma} x\right)}>0, x>0$,

(j) $\rho_{A}=-\frac{v^{\prime \prime}(x)}{v^{\prime}(x)}=\frac{v^{\prime \prime}(-x)}{v^{\prime}(-x)}<0, x<0$,

(k) $\rho_{R}=-\frac{x v^{\prime \prime}(x)}{v^{\prime}(x)}=\frac{(-x) v^{\prime \prime}(-x)}{v^{\prime}(-x)}<0, x<0$,

Corollary 1 : From (f) and (g) of Proposition 31, we get that $\epsilon_{v}(x) \rightarrow \sigma$ as $x \downarrow 0$ and as $x \uparrow 0$. Hence, $\epsilon_{v}(x)$ is defined for all $x \in(-\infty, \infty), \epsilon_{v}(x)=\sigma+\frac{1-\gamma}{1+\frac{\gamma}{\theta|x|}}>0, \epsilon_{v}(x)$ is increasing in $|x|$ and $\epsilon_{v}(x) \rightarrow \sigma+1-\gamma \leq 1$, as $|x| \rightarrow \infty$. 
Remark 1 (SIE value function): In the light of Corollary 1, we may call the value function (3.11) a simple increasing elasticity (SIE) value function.

\section{Explaining the anomalies}

Here, we put together the results of sections 2 and 3. In (4.1), (4.3) and (4.4), below, we reproduce the discount functions (2.12), (2.14) and (2.15) but with the parameter restrictions implied by the propositions of section 2 . For completeness, we also reproduce the PPL-discount function (2.13), with the relevant parameter restrictions, as (4.2).

$$
\begin{aligned}
& \text { exponential: } D_{ \pm}(r, t)=e^{-\beta_{ \pm}(t-r)}, 0<\beta_{-}<\beta_{+} \text {, } \\
& \text { PPL : } \quad D_{ \pm}(0,0)=1, \\
& D_{ \pm}(0, t)=e^{-\left(\delta_{ \pm}+\beta_{ \pm} t\right)}, t>0, \\
& D_{ \pm}(r, t)=e^{-\beta_{ \pm}(t-r)}, 0<r \leq t, \\
& \text { where } 0<\beta_{-} \leq \beta_{+}, 0<\delta_{-} \leq \delta_{+} \\
& \text {and either } \beta_{-}<\beta_{+} \text {or } \delta_{-}<\delta_{+} \text {. } \\
& \mathbf{L P}: D_{ \pm}(r, t)=\left(\frac{1+\alpha_{ \pm} t}{1+\alpha_{ \pm} r}\right)^{-\frac{\beta_{ \pm}}{\alpha \pm}} \\
& 0<\alpha_{-} \leq \alpha_{+}, 0<\frac{\beta_{-}}{\alpha_{-}} \leq \frac{\beta_{+}}{\alpha_{+}} \\
& \text {and either } \alpha_{-}<\alpha_{+} \text {or } \frac{\beta_{-}}{\alpha_{-}}<\frac{\beta_{+}}{\alpha_{+}} \text {. }
\end{aligned}
$$


The reasons for the parameter restrictions in (4.1) to (4.4) are as follows:

Exponential (4.1): $\beta_{ \pm}>0$ ensures that $D_{ \pm}(s, t)$ is strictly increasing in $s$ and strictly decreasing in $t$. $\beta_{-}<\beta_{+}$ensures that $D_{+}(0, t)<D_{-}(0, t)$ for $t>0$, as required by Proposition 29.

PPL (4.2): $0<\beta_{-} \leq \beta_{+}, 0<\delta_{-} \leq \delta_{+}$ensures that $D_{ \pm}(s, t)$ is strictly increasing in $s$ and strictly decreasing in $t$. They also ensure that A1, gain-loss asymmetry, is satisfied. If either $\beta_{-}<\beta_{+}$or $\delta_{-}<\delta_{+}$, then A3, the common difference effect, is also satisfied.

LP (4.3): $\alpha_{ \pm}>0$ and $\beta_{ \pm}>0$ ensure that $D_{ \pm}(r, t)$ is strictly increasing in $r$ and strictly decreasing in $t . \alpha_{-} \leq \alpha_{+}$and $\frac{\beta_{-}}{\alpha_{-}} \leq \frac{\beta_{+}}{\alpha_{+}}$(at least one of them being strict) ensure that $D_{+}(0, t)<D_{-}(0, t)$ for $t>0$, as required by Proposition 29 .

RS (4.4): $\alpha_{ \pm}>0, \beta_{ \pm}>0, \tau>0$ and $\rho>0$ ensure that $D_{ \pm}(r, t)$ is strictly increasing in $r$ and strictly decreasing in $t . \alpha_{-} \leq \alpha_{+}, \frac{\beta_{-}}{\alpha_{-}} \leq \frac{\beta_{+}}{\alpha_{+}}$(at least one of them being strict), $\tau_{+}=\tau_{-}=\tau$ and $\rho_{+}=\rho_{-}=\rho$ ensure that $D_{+}(0, t)<D_{-}(0, t)$ for $t>0$, as required by Proposition 29. $\rho \leq 1$ ensures subadditivity (Proposition 8 (a) and (b)). $\tau \rho \leq 1$ is required to ensure that $\gamma$-delay is satisfied (with $\gamma=\tau \rho$ ) and, hence, A3. ${ }^{22}$

Generalized RS (4.5): The restrictions guarantee that (4.5) is a continuous discount function in the sense of Definition 2 and that $D_{+}(0, t)<D_{-}(0, t)$ in compliance with Proposition 29.

For ease of reference, we reproduce (3.11) below.

$$
\begin{aligned}
v(x) & =\frac{\gamma x^{\sigma}}{1-\gamma}\left(\mu+\frac{\theta}{\gamma} x\right)^{1-\gamma}, x \geq 0, \\
v(x) & =-\lambda v(-x) \\
& =-\lambda \frac{\gamma(-x)^{\sigma}}{1-\gamma}\left(\mu-\frac{\theta}{\gamma} x\right)^{1-\gamma}, x<0, \\
\mu & >0, \theta>0, \lambda>1,0<\sigma \leq \gamma<1,
\end{aligned}
$$

Proposition 33 : Each of the four discount functions (4.2), (4.3), (4.4) and (4.5), when combined with the SIE value function (4.6), satisfies assumptions A0 to A6, i.e., all the assumptions.

\subsection{A summing up}

To sum up so far, exponential discounting (4.1) (even with different discount rates for gains and losses) cannot explain the common difference effect (but see subsections 5.2 and 5.3 , below). On the other hand, the PPL-discount function (4.2), the LP-discount function (4.3) and the RS-discount function (4.4) can all explain the common difference effect. But

\footnotetext{
${ }^{22}$ To see why we need $\tau_{+}=\tau_{-}$, suppose $0<\tau_{+}<\tau_{-}<1$. If $t>1$, then $t^{\tau_{+}}<t^{\tau_{-}}$. But if $0<t<1$, then $t^{\tau_{+}}>t^{\tau_{-}}$. For the same reason, we need $\rho_{+}=\rho_{-}$.
} 
they explain it in different ways. The PPL (4.2) and LP (4.3) discount functions explain the common difference effect with declining impatience. For PPL, there is a sudden drop in impatience from time $t=0$ to times $t>0$, with impatience being constant for all times $t>0$. For LP, on the other hand, the decline in impatience is continuous (recall Example 3). By contrast, the RS-discount function (4.4), on account of its subadditivity (for $0<\rho \leq 1$ ) can explain the common difference whether we have declining impatience $(0<\tau<1)$, constant impatience $(\tau=1)$ or increasing impatience $(\tau>1)$, provided $\tau \rho \leq 1$ (recall Proposition 8 and Example 3).

On the other hand, none of the discount functions (4.1), (4.2) or (4.3) can explain (apparent) intransitive preferences such as that exhibited by (2.24), recall Proposition 7 and subsection 2.8 .

Thus, it emerges that of discount functions (4.1), (4.2), (4.3) and (4.4), the RS-discount function (4.4) is the most satisfactory because, when combined with the SIE value function, reference time/wealth, and different discount functions for gains and losses, it can explain all the anomalies: gain-loss asymmetry, the magnitude effect, the common difference effect, delay-speedup asymmetry as well as subadditivity and (apparent) intransitivity.

Finally, we give an example that illustrates the difficulty inherent in taking the discount function for gains to be same as that for losses.

Example 4 : (Identical discount functions for gains and losses): For our illustration, we take $\mu=1, \theta=\frac{1}{2}, \lambda=2,0<\sigma=\gamma=\frac{1}{2}$. These satisfy the restrictions on the value function parameters in (3.11). Take $\alpha_{-}=\alpha_{+}=1, \beta_{-}=1, \beta_{+}=2, \rho=\tau=1$. These satisfy the restrictions on the discount functions parameters in (4.3) and (4.4). With these parameter values, the SIE value function (3.11) takes the form:

$$
\begin{aligned}
& v(x)=x^{\frac{1}{2}}(1+x)^{\frac{1}{2}}, x \geq 0, \\
& v(x)=-2 v(-x)=-2(-x)^{\frac{1}{2}}(1-x)^{\frac{1}{2}}, x<0,
\end{aligned}
$$

The LP (4.3) and RS (4.4) discount functions take the forms:

$$
\begin{aligned}
\boldsymbol{L P} & : \quad D_{+}(r, t)=(1+r)^{2}(1+t)^{-2}, \\
D_{-}(r, t)= & (1+r)(1+t)^{-1}, \\
\boldsymbol{R S}: \quad & D_{+}(r, t)=(1+t-r)^{-2}, r \leq t, \\
D_{-}(r, t)= & (1+t-r)^{-1}, r \leq t .
\end{aligned}
$$

Note that for both the LP and RS-discount functions, we have:

$$
\begin{array}{cl}
\boldsymbol{L P} \& \boldsymbol{R S}: & D_{+}(0, t)=(1+t)^{-2}, t \geq 0, \\
D_{-}(0, t)= & (1+t)^{-1}, t \geq 0,
\end{array}
$$


Let $V_{0}(x, t)$ be the value of $x$ received at time $t$, discounted back to time 0 . Then (2.9), (4.7), (4.8), (4.13) and (4.14) give:

$$
\begin{aligned}
& V_{0}(x, t)=x^{\frac{1}{2}}(1+x)^{\frac{1}{2}}(1+t)^{-2}, x \geq 0, \\
& V_{0}(x, t)=-2(-x)^{\frac{1}{2}}(1-x)^{\frac{1}{2}}(1+t)^{-1}, x<0 .
\end{aligned}
$$

Rewrite (4.15) and (4.16) as:

$$
\begin{aligned}
& V_{0}(x, t)=x^{\frac{1}{2}}(1+x)^{\frac{1}{2}}(1+t)^{-2}, x \geq 0, \\
& V_{0}(x, t)=-2(1+t)(-x)^{\frac{1}{2}}(1-x)^{\frac{1}{2}}(1+t)^{-2}, x<0 .
\end{aligned}
$$

Comparing (4.17) and (4.18), we see that gains and losses now have the same discount function, $D(0, t)=(1+t)^{-2}$. But now the parameter of loss aversion, $\lambda(0, t)=2(1+t)$, depends on the time, $t$, when $x$ is received. In particular, $\lambda \rightarrow \infty$ as $t \rightarrow \infty$. We know of no empirical evidence that supports this.

\section{Alternatives and extensions}

In this section, we compare the reference-time theory (RT) of section 2 with four recent developments.

First, we consider the tradeoff model of intertemporal choice of Scholten and Read (2006b), SR for short. We will argue that SR's tradeoff criterion can be represented by a discount function. Hence, it can be incorporated within RT. The gain is that their psychological arguments for their tradeoff model give support for RT theory and, in particular, their own RS-discount function.

The second development we consider is Halevy (2007), $\mathrm{H}$ for short, who shows that the common difference effect is compatible with exponential discounting, provided subjects are non-expected utility maximizers and exhibit the certainty effect. The certainty effect was first proposed as an explanation of the Allais paradox: subjects are much more sensitive

to a change from certainty to uncertainty than they are to changes in the middle range of probabilities.

The third is the theory of vague time preferences of Manzini and Mariotti (2006), MM for short. Again, they can explain the common difference effect without departing from exponential discounting. However, we believe that the importance of $\mathrm{H}$ and $\mathrm{MM}$ far transcends their ability to explain the common difference effect. On the other hand, and because in their present formulations they do not include any reference dependence, they are unable to explain gain-loss asymmetry, delay-speedup asymmetry, subadditivity and (apparent) intransitivity. By contrast, RT theory can explain all the anomalies. Nevertheless, we believe that it is desirable, and easy, to extend RT theory to incorporate 
uncertainty, as in $\mathrm{H}$, and multiple criteria, as in MM. We show this, below, in the context of simple examples.

The fourth recent development we discuss here is the theory of intransitive preferences and relative discounting of Ok and Masatlioglu (2007), OM for short. This is the most radical of all the theories considered so far. From the outset it neither assumes transitivity nor additivity and, hence, is compatible with these two phenomena. In its present formulation, it cannot account for either gain-loss asymmetry or delay-speedup asymmetry. Furthermore, the lack of transitivity will make it hard to work with this theory, as the authors themselves explain. On the other hand, these problems can all be resolved in the special case of a transitive preference relation. But then their model becomes additive. In this case, OM would reduce to a standard discounting model.

Finally, all five theories (SR, H, MM, OM and RT) can explain the magnitude effect, when combined with the SIE value function developed in this paper.

\subsection{The tradeoff model of intertemporal choice}

Read and Scholten's critique of discounting models, including their own, led them to develop their tradeoff model of intertemporal choice (Read and Scholten, 2006). It is worth quoting their abstract in full:

"Research on intertemporal judgement and choices between a smaller-sooner and a larger-later outcome has revealed many anomalies to the discountedutility model. Attempts to account for these anomalies within the discounting paradigm have resulted in convoluted and psychologically opaque models. We therefore develop a new model of intertemporal choice, the tradeoff model, in which choice results from a tradeoff between the perceived time difference (interval) and the perceived outcome difference (compensation). This model is both more parsimonious and more intuitive than any rival discounting model of comparable scope. Moreover, it accurately describes archival data as well as data from new experiments."

We argue that the tradeoff model of Scholten and Read (2006b) can be incorporated within RT-theory. If this is accepted, then their tradeoff model lends further support to the RT-theory and, in particular, their own discount function (2.15) and its generalization (5.13), below.

We proceed by first recasting their model in a more general form (and indicate how their model is to be obtained as a special case). However, there should be no presumption that they would agree with our reformulation. They develop their model through three successive versions. We concentrate on their fourth and final version, page 15. 
Let $r \geq 0$ be the reference point for time. ${ }^{23}$ The tradeoff model establishes preference relationships, $\prec_{r}$ and $\sim_{r}$ between outcome pairs $(x, s)$ and $(y, t)$. Thus $(x, s) \prec_{r}(y, t)$ if, and only if, $y$ received at time $t$ is strictly preferred to $x$ received at time $s$. Similarly, $(x, s) \sim_{r}(y, t)$ if, and only if, $y$ received at time $t$ is equivalent to $x$ received at time $s$. These relationship are established using three functions, a value function, $u$, a tradeoff function $Q$ and a delay-perception function, $w$. We make the following assumptions: $Q$ : $[0, \infty) \stackrel{\text { onto }}{\longrightarrow}[0, \infty)$ is strictly increasing, $w:[0, \infty) \stackrel{\text { onto }}{\longrightarrow}[0, \infty)$ is strictly increasing (the same as in (5.13), below). ${ }^{24}$

First, let $x>0, y>0$ and $s \geq r \geq 0, t \geq r$. Then:

$$
\begin{aligned}
& (x, s) \sim r(y, t) \Leftrightarrow Q[w(t)-w(r)]-Q[w(s)-w(r)]=u(y)-u(x), \\
& (x, s) \prec r(y, t) \Leftrightarrow Q[w(t)-w(r)]-Q[w(s)-w(r)]<u(y)-u(x) .
\end{aligned}
$$

Second, let $x<0, y<0$ and (as before) $s \geq r \geq 0, t \geq r$. Then:

$$
\begin{aligned}
& (x, s) \sim r(y, t) \Leftrightarrow Q(w(t)-w(r))-Q(w(s)-w(r))=u(x)-u(y), \\
& (x, s) \prec r(y, t) \Leftrightarrow Q(w(t)-w(r))-Q(w(s)-w(r))>u(x)-u(y) .
\end{aligned}
$$

For completeness, we also need (again, $s \geq r \geq 0, t \geq r$ ):

$$
\begin{aligned}
(0, s) & \sim{ }_{r}(0, t), \\
x & <0 \Rightarrow(x, s) \prec_{r}(0, t), \\
y & >0 \Rightarrow(0, s) \prec_{r}(y, t), \\
x & <0, y>0 \Rightarrow(x, s) \prec_{r}(y, t) .
\end{aligned}
$$

To get the tradeoff model of Read and Scholten, set $r=s$ in the above equations. ${ }^{25}$ To define a discount function, $D$, that expresses these preferences, let

$$
\begin{aligned}
& v(x)=e^{u(x)}, \text { for } x>0, \\
& v(x)=-e^{-u(x)}, \text { for } x<0 .
\end{aligned}
$$

Then all the above relations, (5.1) to (5.8), can be summarized by the following. For all $x, y$ and all $r, s, t$ such that $s \geq r \geq 0, t \geq r$ :

$$
\begin{aligned}
& (x, s) \quad \sim \quad r(y, t) \Leftrightarrow v(x) e^{-Q[w(s)-w(r)]}=v(y) e^{-Q[w(t)-w(r)]}, \\
& (x, s) \quad \prec \quad r(y, t) \Leftrightarrow v(x) e^{-Q(w(s)-w(r))}<v(y) e^{-Q[w(t)-w(r)]} .
\end{aligned}
$$

\footnotetext{
${ }^{23}$ To ease the burden of notation, we shall suppress reference to the reference point for wealth, $w_{0}$. Thus, in what follows, we write $\prec_{r}$ and $\sim_{r}$ when we should have written $\prec_{r, w_{0}}$ and $\sim_{r, w_{0}}$, respectively.

${ }^{24}$ They explicitly state two assumptions: $Q^{\prime}>0, Q^{\prime \prime}<0$. However, in the next paragraph, they say that $Q^{\prime \prime}>0$ for sufficiently small intervals. So, we make no assumptions on $Q^{\prime \prime}$. They explicitly state no further assumptions on $Q$ and $w$. However, we believe our other assumptions on $Q$ and $w$ are in line with what they intend (see their equations (2) and (5) for the earlier, and simpler, versions of their model).

${ }^{25}$ They explicitly state only (5.1) and (5.3) (with $r=s$ ). However, we believe that our other equations are in line with their framework.
} 
(5.11) and (5.12) suggest we take our discount function to be

$$
\begin{aligned}
& \text { generalized RS: } D(r, t)=e^{-Q[w(t)-w(r)]}, 0 \leq r \leq t, \\
& Q \quad: \quad[0, \infty) \stackrel{\text { onto }}{\longrightarrow}[0, \infty) \text { is strictly increasing, } \\
& w: {[0, \infty) \stackrel{\text { onto }}{\longrightarrow}[0, \infty) \text { is strictly increasing. } }
\end{aligned}
$$

which is a generalization of the discount function (2.15) of Scholten and Read (2006a).

Thus, RT-theory can incorporate the tradeoff model.

\subsection{The certainty effect}

A test of a theory $(\mathrm{T})$ is always a test of $\mathrm{T}$ plus auxiliary assumptions (O). Thus, a refutation of $\mathrm{T} \& \mathrm{O}$ may be a refutation of $\mathrm{O}$ rather than $\mathrm{T}$. However, since $\mathrm{O}$ is often left implicit, a refutation of T\&O may be misconstrued as a refutation of $\mathrm{T}$ rather than $\mathrm{O}$. A case in point may be $\mathrm{T}=$ 'exponential discounting' and $\mathrm{O}=$ 'uncertainty is not relevant'. In testing the common difference effect, not only is it better if subjects are paid 'real money', the delays should be realistic too, i.e., quite long. Despite the experimenters' best efforts to eliminate uncertainty, there will always be a residual risk that the subjects will not receive their promised payoffs. If subjects were expected utility (EU) maximizers, then risk would not matter (Example 5, below). However, if subjects overweight low probabilities and underweight high probabilities (as in many non-EU theories), then risk matters (Example 6, below). Moreover, the lower the residual risk the greater will be its effect! (Example 7, below.) ${ }^{26}$ Thus, Halevy (2007) argues that the common difference effect may, in fact, be a refutation of EU rather than exponential discounting.

The above points are illustrated by the following three examples. They all involve a choice between receiving $\$ 1000$ now or $\$ 1100$ next year and, simultaneously, a choice between receiving these two sums 10 and 11 years from now, respectively. We use the SIE value function (4.6), so that

$$
v(1000)=1000.5 \text { and } v(1100)=1100.5 \text {. }
$$

Let the discount function be $D(s, t)$ and the probability weighting function be $w(p)$, where $p$ is the probability that the payoff will actually be paid one year from now. We assume independence across years so that the probability of receiving the payoff $t$ years from now is $p^{t}$. Let $(x, t)$ be the event $\$ x$ is received in year $t$ and let $(x, s) \prec(y, t)$ mean $(y, t)$ is strictly preferred to $(x, s)$. We take the current level of wealth, $w_{0}$, and present time, $r=0$, to be the reference points for wealth and time, respectively (and, to simplify notation, we

\footnotetext{
${ }^{26}$ So, experimenters, by doing their best to reduce residual risk with the aim of getting a sharper refutation of exponential discounting are, actually, achieving the opposite.
} 
have dropped the subscripts, $w_{0}, r$, from $\left.\prec_{w_{0}, r}\right)$. We thus have:

No common difference effect: $(1100,1) \prec(1000,0) \Rightarrow(1100,11) \prec(1000,10)$

$$
(1100,1) \prec(1000,0) \Leftrightarrow v(1100) D(0,1) w(p)<v(1000),
$$

$$
(1100,11) \prec(1000,10) \Leftrightarrow v(1100) D(0,11) w\left(p^{11}\right)<v(1000) D(0,10) w\left(p^{10}\right) .
$$

Example 5 (exponential discounting with expected utility): Assume exponential discounting, so $D(s, t)=e^{-\beta(t-s)}, \beta>0$, and expected utility, so $w(p)=p$. Substitute in (5.16), (5.17) to get:

$$
\begin{gathered}
(1100,1) \prec(1000,0) \Leftrightarrow v(1100) e^{-1} p<v(1000), \\
(1100,11) \prec(1000,10) \Leftrightarrow v(1100) e^{-11 \beta} p^{11}<v(1000) e^{-10 \beta} p^{10},
\end{gathered}
$$

(5.19) is equivalent to:

$$
(1100,11) \prec(1000,10) \Leftrightarrow v(1100) e^{-1} p<v(1000)
$$

From (5.18) and (5.20) we see that $(1100,1) \prec(1000,0) \Leftrightarrow(1100,11) \prec(1000,10)$. Thus, exponential discounting together with expected utility ${ }^{27}$ imply no common difference effect. Hence, the observation of a common difference effect is a rejection of the joint hypothesis of exponential discounting and expected utility. Thus, it would imply the rejection of one or the other (or both) but not, necessarily, exponential discounting.

Example 6 (Exponential discounting with non-expected utility): We take cumulative prospect theory (Tversky and Kahneman, 1992) as our model of non-expected utility. Take $D(r, t)=e^{-\beta(t-r)}, \beta=0.04$, and $w(p)=e^{-(-\ln p)^{\alpha}}$ (the Prelec probability weighting function $)^{28}$ with $\alpha=0.5$. Let $p=0.98$. Substitute in (5.16), (5.17), using (5.14), to get:

$$
\begin{gathered}
(1100,1) \prec(1000,0) \Leftrightarrow 1100.5 e^{-0.04} e^{-(-\ln 0.98)^{0.5}}<1000.5 \\
(1100,11) \prec(1000,10) \Leftrightarrow 1100.5 e^{-0.44} e^{-\left(-\ln (0.98)^{11}\right)^{0.5}}<1000.5 e^{-0.4} e^{-\left(-\ln (0.98)^{10}\right)^{0.5}} .
\end{gathered}
$$

A calculation shows that the inequality of the right hand side of (5.21) holds while the corresponding inequality in (5.22) does not hold. Hence, $(1100,1) \prec(1000,0)$ but $(1100,11) \succ(1000,10)$. Thus, exponential discounting may be consistent with an observation of a common difference effect, if subjects do not behave according to expected utility.

\footnotetext{
${ }^{27}$ Or no risk so that $p=1$ and, hence, $w(p)=w(1)=1$.

${ }^{28}$ See Prelec (1998), Luce (2001) and al-Nowaihi and Dhami (2006b).
} 
Example 7 (It's the certainty effect that's doing the work): The same as for Example (6), except that now $p=0.4$, rather than $p=0.98$. Substitute in (5.16), (5.17), using (5.14), to get:

$$
\begin{gathered}
(1100,1) \prec(1000,0) \Leftrightarrow 1100.5 e^{-0.04} e^{-(-\ln 0.4)^{0.5}}<1000.5, \\
(1100,11) \prec(1000,10) \Leftrightarrow 1100.5 e^{-0.44} e^{-\left(-\ln (0.4)^{11}\right)^{0.5}}<1000.5 e^{-0.4} e^{-\left(-\ln (0.4)^{10}\right)^{0.5}} .
\end{gathered}
$$

A calculation shows that the inequality of the right hand side of (5.23) holds and also the corresponding inequality in (5.24). Hence, $(1100,1) \prec(1000,0)$ and $(1100,11) \prec$ $(1000,10)$. Thus, the common difference effect is due to the certainty effect in particular, rather than uncertainty as such.

Example 7 suggests that if the common difference effect is due to the certainty effect alone, rather than a combination of the certainty effect and non-exponential discounting, then the phenomenon should disappear for probabilities around 0.4.

\subsection{Vague time preferences}

Manzini and Mariotti (2006) develop a theory of vague time preferences and discuss the psychological foundations for such an approach. The intuition behind this theory is that the choice between, say, receiving $\$ 1000$ now and $\$ 1100$ next year is clearer than the choice between these two sums received 10 and 11 years from now, respectively. MM propose three criteria to choose between $(x, t)$ and $(y, s)$. The primary criterion is to choose whichever has the highest present utility value. If the two present values are not 'significantly' different, then the subject chooses the one with the highest monetary value (secondary criteria). If they have the same monetary values, so that the secondary criterion fails, then the subject behaves according to the third criterion: 'choose the outcome that is delivered sooner'. If all three criteria fail, then the subject is indifferent. Thus, MM achieve a complete, though intransitive, ordering. In particular, indifference here is not an equivalence relationship. Suppose that two present values are significantly different if their difference is greater than $\sigma$, where $\sigma$ is positive real number. Then we can state these criteria formally as follows. $(x, t) \prec_{w_{0}, r}(y, s)$ if, and only if, one of the following holds ${ }^{29}$ :

1. $v(y) D_{ \pm}(r, s)-v(x) D_{ \pm}(r, t)>\sigma$, or

2. $\left|v(y) D_{ \pm}(r, s)-v(x) D_{ \pm}(r, t)\right| \leq \sigma$, and $x<y$, or

3. $\left|v(y) D_{ \pm}(r, s)-v(x) D_{ \pm}(r, t)\right| \leq \sigma, x=y$ and $s<t$.

\footnotetext{
${ }^{29}$ More generally, $\sigma$ is a 'vagueness function', in which case ' $\left|v(y) D_{ \pm}(r, s)-v(x) D_{ \pm}(r, t)\right| \leq \sigma$ ' is replaced by ' $v(y) D_{ \pm}(r, s)-v(x) D_{ \pm}(r, t) \leq \sigma(x ; r, t)$ and $v(x) D_{ \pm}(r, t)-v(y) D_{ \pm}(r, s) \leq \sigma(y ; r, s)$ '
} 
Obviously, if $x$ and $y$ are vectors, then extra criteria can be added. Present utility values whose difference is less than $\sigma$ are regarded as not significantly different. This could be because, for example, the decision maker is not sure of the appropriate value function or discount function to use. Therefore, the decision maker does not want the decision to depend too critically on the choice of these functions. On the other hand, the decision maker might be absolutely sure that more is better than less and sooner is better than later. Example 8, below, shows how this theory can explain the common difference effect.

Example 8 : Consider the choice between receiving $\$ 1000$ now and $\$ 1100$ next year and the choice between these two sums received 10 and 11 years from now, respectively. As with the examples in subsection 5.2, we use the SIE value function (4.6), so that 5.14 holds. We use the exponential discount function (2.12) with $\beta=0.1$ and the reference time $r=0, D_{ \pm}(0, t)=e^{-0.1 t}$. We take $\sigma=3$, so that present utility values whose difference is less than 3 are regarded as not significantly different. Using these values, we get $v(1000)-v(1100) e^{-0.1}=1000.5-1100.5 e^{-0.1}=4.7264>3$. Hence, the primary criterion holds and the decision maker prefers $\$ 1000$ now to $\$ 1100$ next year. Next, $\left|v(1100) e^{-1.1}-v(1000) e^{-1}\right|=\left|1100.5 e^{-1.1}-1000.5 e^{-1}\right|=1.7388<3$. Hence, the primary criterion fails, and the decision maker considers the second criterion. Since $1000<1100$, the second criterion holds. The decision maker prefers $\$ 1100$ received 11 years from now to $\$ 1000$ received 10 years from now. We have an illustration of the common difference effect.

Recall, from subsection 2.8 above, that the experimental results of Roelofsma and Read (2000) supported 'sooner is better than larger' against 'larger is better than sooner'. However, if the order of the secondary criteria is reversed, so that sooner is better than larger (in agreement with the experimental results of Roelofsma and Reed, 2000), then $\$ 1000$ received 10 years from now would be better than $\$ 1100$ received 11 years from now, and we would not get a common difference effect.

However, whether MM's explanation of the common difference effect is acceptable or not, to us the main contribution of their paper lies in the use of primary and secondary criteria. This appears to us to be a more accurate description of actual decision making compared to the assumption of a single criterion.

\subsection{Intransitive preferences and relative discounting}

Ok and Masatlioglu (2007) (henceforth OM) accommodate (apparent) intransitivity, such as (2.24), by regarding it as real. Thus, they develop a theory of intransitive time preferences. At time 0 , a decision maker has a binary relationship, $\preceq$, on the set $\chi=X \times[0, \infty)$, 
where $X$ is a non-empty set. Let $x, y \in X$ and $s, t \in[0, \infty)$, then $(x, s) \preceq(y, t)$ is to be interpreted as ' $y$ received at time $t$ is (weakly) preferred to $x$ received at time $s$ '.

Let $\sim$ and $\prec$ be the symmetric and asymmetric parts of $\preceq$, respectively. For each $t \in[0, \infty), \preceq_{t}$ is the $t$-th time projection of $\preceq$ onto $X$, i.e., $x \preceq_{t} y$, if, and only if, $(x, t) \preceq(y, t)$. In particular, $\preceq_{0}$ is the projection of $\preceq$ onto $X$ at time 0 (and, similarly, for $\sim_{t}$ and $\prec_{t}$ ).

If $X$ is a metric space, then further structure can be imposed on $\preceq$. In particular (OM, p218):

Definition 14 (time preferences): Let $X$ be a metric space, then $\preceq$ is a time preference on $\chi$ if

(i) $\preceq$ is complete and continuous,

(ii) $\preceq_{0}$ is complete and transitive,

(iii) $\preceq_{t}=\preceq_{0}$ for each $t \in[0, \infty)$.

In Definition 14, note that transitivity is imposed on $\preceq_{0}$ (and, hence, also on $\preceq_{t}$ ) but transitivity is not imposed on $\preceq$. Hence, neither $\prec$ nor $\sim$ are, necessarily, transitive. In particular, $\sim$ is not, in general, an equivalence relationship.

Let $\mathbb{R}$ be the set of real numbers, $\mathbb{R}_{+}$the set of non-negative reals and $\mathbb{R}_{++}$the set of positive reals. Recall that a homeomorphism is a mapping that is $1-1$, onto, continuous and its inverse is also continuous. Then

Proposition 34 (OM, Theorem 1) ${ }^{30}$ : Let $X$ be an open interval in $\mathbb{R}$ and $\preceq$ a binary relation on $\chi$. $\preceq$ is a time preference on $\chi$ that satisfies properties (A1)-(A6) if, and only, there exit an increasing homeomorphism $U: X \rightarrow \mathbb{R}_{++}$and a continuous map $D: \mathbb{R}_{+}^{2} \rightarrow \mathbb{R}_{++}$such that, for all $x, y \in X$ and $s, t \in[0, \infty)$,

$$
(x, s) \preceq(y, t) \text { iff } U(x) \leq U(y) D(s, t),
$$

while (i) for given $s, D(s, t)$ is decreasing in $t$ with $D(s, \infty)=0$, and (ii) $D(t, s)=$ $1 / D(s, t)$.

Suppose $s \leq t$. Then (5.25) says that $y$ received at time $t$ is (weakly) preferred to $x$ received at time $s$ if, and only if, the (undated) utility of $x$ is less or equal to the (undated) utility of $y$ discounted from time $t$ back to time $s$ by the discount factor $D(s, t)$. In this case, part (i) of Proposition 34 implies the following. Fix the time, $s$, at which $x$ is received. Let the time, $t$, at which $y$ is received, recede into the future. Then the value of the utility

\footnotetext{
${ }^{30}$ Assumption A1 to A6 of OM are plausible and clearly stated. We do not reproduce them here because we only need the theorems that OM derive from them. Also note that $D(s, t)$ here corresponds to $\eta(t, s)$ in $\mathrm{OM}$.
} 
of $y$, discounted back to time $s$, decreases. In the limit, as the receipt of $y$ is indefinitely postponed, the value of its utility, discounted back to time $s$, approaches zero. Part (ii) of Proposition 34 says that compounding forward, from time $s$ to time $t$, is the inverse of discounting backwards from time $t$ to time $s$.

For each $r \in[0, \infty)$, let $\preceq^{r}$ be the restriction of $\preceq$ to $X \times[r, \infty)$, i.e., to times $t \geq r$. Thus, for $r \leq s$ and $r \leq t,(x, s) \preceq^{r}(y, t)$ if, and only if, $(x, s) \preceq(y, t)$.

We can now point to the main differences between RT and OM.

First, note that $U$ in Proposition 34 can take only positive values while $v$ in (2.4)(2.5) takes both positive and negative values. ${ }^{31}$ To bypass this problem, we consider only the domain of strictly positive gains. Let $w_{0}$ be the reference point for wealth. Take $X=\left\{w-w_{0}: w>w_{0}\right\}=(0, \infty)$ and let $\preceq$ satisfy the conditions of Proposition 34. Let $(U, D)$ be the representation of $\preceq$ guaranteed by that Proposition. ${ }^{32}$

From subsection 2.3 recall that, for each $r \in[0, \infty), \preceq_{w_{0}, r}$ is a complete transitive order on $(-\infty, \infty) \times[r, \infty)$ and, hence, also on $X \times[r, \infty)$. The second point we wish to make is that, in general, $\preceq_{w_{0}, r}$, unlike $\preceq^{r}$, is not the restriction to $X \times[r, \infty)$ of some complete binary relationship on $X \times[0, \infty)$. Thus OM and RT are not compatible and neither is a special case of the other.

Third, $\preceq_{w_{0}, r}$ is transitive while, in general, $\preceq^{r}$ is not transitive. To elaborate this point, consider $(x, r),(y, s)$ and $(z, t)$, where $x, y, z \in X$ and $s, t \in[r, \infty)$. Suppose $(x, r) \preceq_{w_{0}, r}(y, s)$ and $(y, s) \preceq_{w_{0}, r}(z, t)$. Since $\preceq_{w_{0}, r}$ is transitive, we can conclude that $(x, r) \preceq_{w_{0}, r}(z, t)$. Now, suppose that $(x, r) \preceq^{r}(y, s)$ and $(y, s) \preceq^{r}(z, t)$. Since $\preceq^{r}$ is not, in general, transitive, we cannot conclude that $(x, r) \preceq^{r}(z, t) \cdot{ }^{33}$ More generally, given a compact subset $C \subset X \times[r, \infty)$, there is no guarantee in OM that it has a maximum under $\preceq^{r}$ (i.e., an $m \in C$ such that $c \preceq^{r} m$ for all $c \in C$ ). This, obviously, will cause great difficulty for any economic theory formulated in the OM framework. On the other hand, in RT theory, and if $D$ is continuous, $C$ will always have a maximum under $\preceq_{w_{0}, r}$.

Fourth, and finally, these problems with OM can all be resolved in the special case where $\preceq$ is transitive. But then $\preceq$ would also be additive. In this case, OM would reduce to the standard discounting model.

\footnotetext{
${ }^{31}$ Hence, in its present formulation, OM cannot explain gain-loss asymmetry. However, it can explain the magnitude effect using the SIE value function (4.6).

${ }^{32}$ Two alternatives are possible. The first is to extend $U$ to a function on $(-\infty, \infty)$ as follows. Define $U(0)=0$ and, for $x<0, U(x)=-\lambda U(-x)$. However, because of the separability assumption in OM, $D_{-}=D_{+}$. The theory would then not be able to explain gain-loss asymmetry. The second alternative is to work with two representations: $\left(U_{+}, D_{+}\right)$for gains and $\left(U_{-}, D_{-}\right)$for losses.

${ }^{33}$ As OM clearly explain, it is for this reason that we should think of $D(s, t)$ in their theory as the relative discount function between times $s$ and $t$.
} 


\section{Summary and conclusions}

The exponential discounting model is known to be subject to a range of anomalies. We try to explain the 6 most important ones in one unified model. These anomalies are gain-loss asymmetry, magnitude effect, common difference effect, delay-speedup asymmetry, nonadditivity of time discounting and apparent intransitivity of time preferences. Furthermore, we show how recent work on intertemporal choice can be incorporated within our model. Our unified model builds on the seminal works of Lowenstein and Prelec (1992) (LP); Phelps and Pollak (1968) and Laibson (1997) (PPL); Roelofsma and Read (2000), Read (2001) and Scholten and Read (2006a) (RRS). We follow LP in taking prospect theory (Kahneman and Tversky, 1979 and Tversky and Kahneman, 1992) as our underlying decision theory. However, we depart from LP in having a reference point for time as well as a reference point for wealth. We also allow for different discount functions for gains and losses. We call our unified theory, the reference-time theory of intertemporal choice, RT, for short (section 2).

LP showed that a value function that can explain the magnitude effect must exhibit increasing elasticity. We show that this is incompatible with several popular classes of value functions including CARA (constant absolute risk aversion), CRRA (constant relative risk aversion), HARA (hyperbolic absolute risk aversion), logarithmic and quadratic. We develop a scheme for generating value functions that exhibit increasing elasticity, as required to explain the magnitude effect. We call the simplest class that has this property the class of simple increasing elasticity value functions (SIE). Each member of this class is formed by a product of a HARA function and a CRRA function and, therefore, is quite tractable (sections 3 and 4 ).

LP explained the gain-loss asymmetry (also known as the sign effect) by assuming different elasticities of the value function for gains and losses. We show that this implies a variable coefficient of loss aversion, in particular, a coefficient of loss aversion that is increasing with time (Example 4, section 4). We depart from LP in assuming constant loss aversion, which is consistent with the evidence. We explain gain loss asymmetry by assuming different discount functions for gains and losses.

LP provided an axiomatic derivation of their generalized hyperbolic discount function (which we called the LP-discount function). For this, they added the extra assumption of linear delay to that of the common difference effect (also known as preference reversal or the delay effect). While there is considerable empirical evidence for the common difference effect, the assumption of linear delay is added purely for convenience. We extended their work as follows. At the most general level, which requires neither linear delay nor the common difference effect, we established our Representation Theorem 2 (Proposition 12). Given an arbitrary delay function (Definition 7), Representation Theorem 2 characterizes 
all continuous discount functions with that delay function. We introduced a weaker notion of subadditivity, which we called $\alpha$-subadditivity (Definition 12). According to our Characterization Theorem 4 (Proposition 21), preferences exhibit the common difference effect if, and only if, $\alpha$-subadditivity holds. We also introduce a generalization of the concept of linear delay of LP. We called this $\gamma$-delay. Our Proposition 23 then showed that $\gamma$-delay implies the common difference effect. Using $\gamma$-delay, we derived the RS-discount function as an $f$-extension for a suitable function, $f$ (Proposition 25). On the other hand, imposing additivity, as well as $\gamma$-delay, gives our Proposition 24. The special case of the latter with $\gamma=1$ gives the LP-discount function. In particular, as with RRS, we can explain the common difference effect as due to either declining impatience, subadditivity or a combination of both. However, our approach is more general because RT can also explain the common difference effect as due to the presence of a small amount of irremovable uncertainty, as in Halevy (2007), or as a consequence of multiple decision criteria, as in Manzini and Mariotti (2006) (see section 5). Thus, RT can accommodate all the known explanations of the common difference effect. In the spirit of RRS, we leave it to the empirical evidence to select the correct explanation. We also showed that delay-speedup asymmetry follows from our other assumptions (Proposition 30).

We showed how the RT theory can be extended to incorporate the attribute model of Scholten and Read (2006b) (section 5). Also in section 5, we compared and contrasted RT theory with Ok and Masatlioglu's (2007) model of intransitive time preferences. We showed that neither is a special case of the other. However, of the two, we believe that the RT theory of this paper is the more tractable theory.

Work over the last two decades has shown the importance of each of the following elements in explaining intertemporal choice: (1) Prospect theory and hyperbolic discounting, as in Lowenstein and Prelec (1992). (2) The interval discount function, as in Scholten and Read (2006a). (3) Multiple criteria, on the lines of Manzini and Mariotti (2006). (4) Uncertainty, as in Halevy (2007). We hope that this paper has shown how to incorporate all these elements, along with a reference point for time and an SIE value function, into a coherent and tractable model, which we called reference-time theory (RT).

\section{Appendix: Proofs}

The proofs of Propositions 26 and 27 are essentially adaptations of those in Loewenstein and Prelec (1992) to the model of this paper.

Proof of Proposition 1: Let $r \in[0, \infty)$ and $t \in[r, \infty)$. Let $\left\{t_{n}\right\}_{n=1}^{\infty}$ be a sequence in $[r, \infty)$ converging to $t$. We want to show that $\left\{D\left(r, t_{n}\right)\right\}_{n=1}^{\infty}$ converges to $D(r, t)$. It is sufficient to show that any monotone subsequence of $\left\{D\left(r, t_{n}\right)\right\}_{n=1}^{\infty}$ converges to $D(r, t)$. In particular, let $\left\{D\left(r, t_{n_{i}}\right)\right\}_{i=1}^{\infty}$ be a decreasing subsequence of $\left\{D\left(r, t_{n}\right)\right\}_{n=1}^{\infty}$. 
Since $\left\{D\left(r, t_{n_{i}}\right)\right\}_{i=1}^{\infty}$ is bounded below by $D(r, t)$, it must converge to, say, $q$, where $D(r, t) \leq q \leq D\left(r, t_{n_{i}}\right)$, for all $i$. Since $D$ is onto, there is a $p \in[r, \infty)$ such that $D(r, p)=q$. Moreover, $t_{n_{i}} \leq p \leq t$, for each $i$. Suppose $D(r, t)<q$. Then $t_{n_{i}}<p$, for each $i$. Hence also $t_{n_{i}}<t$, for each $i$. But this cannot be, since $\left\{t_{n_{i}}\right\}_{i=1}^{\infty}$, being a subsequence of the convergent sequence $\left\{t_{n}\right\}_{n=1}^{\infty}$, must also converge to the same limit, $t$. Hence, $D(r, t)=q$. Hence, $\left\{D\left(r, t_{n_{i}}\right)\right\}_{i=1}^{\infty}$ converges to $D(r, t)$. Similarly, we can show that any increasing subsequence of $\left\{D\left(r, t_{n}\right)\right\}_{n=1}^{\infty}$ converges to $D(r, t)$. Hence, $\left\{D\left(r, t_{n}\right)\right\}_{n=1}^{\infty}$ converges to $D(r, t)$. Hence, $D(r, t)$ is continuous in $t$.

Proof of Proposition 2: It is straightforward to check that each of (2.12), (2.14), (2.15) and (5.13) is a continuous discount function in the sense of Definition 2. It is also straightforward to check that $(2.13)$ is a discount function. The reason the latter is not a continuous discount function is that $\lim _{t \rightarrow 0+} D(0, t)<D(0,0)$.

Proof of Proposition 3 (Time sensitivity): Let $D(r, t)$ be a continuous discount function and $r \geq 0$. Suppose $0<x \leq y$. From (2.4) and (2.5), it follows that $0<v(x) \leq$ $v(y)$ and, hence, $0<\frac{v(x)}{v(y)} \leq 1$. Since, by Definition $2(\mathrm{i}), D(r, t):[r, \infty) \stackrel{\text { onto }}{\rightarrow}(0,1]$, it follows that $\frac{v(x)}{v(y)}=D(r, t)$ for some $t \in[r, \infty)$. A similar argument applies if $y<x<0$.

Proof of Proposition 4 (Existence of present values): Let $r \leq t$ and $y \geq 0$. Then, $0<D(r, t) \leq 1$. Hence, $0=v(0) \leq v(y) D(r, t) \leq v(y)$. Since $v$ is continuous and strictly increasing, it follows that $v(y) D(r, t)=v(x)$ for some $x \in[0, y]$. Similarly, if $y \leq 0$, then $v(y) D(r, t)=v(x)$ for some $x \in[y, 0]$.

Proof of Proposition 5: (a) Suppose that $D(r, t)=[\varphi(r)]^{-1} \varphi(t)$ for some strictly decreasing real valued function, $\varphi:[0, \infty) \rightarrow(0,1]$. We first check that $D$ satisfies all parts of Definition 2. Clearly, $D(r, t)$ is strictly decreasing in $t$ and strictly increasing in $r$. Let $r \in[0, \infty) . D(r, r)=[\varphi(r)]^{-1} \varphi(r)=1$. Hence, for fixed $r \in[0, \infty), t \mapsto D(r, t)$ maps $[r, \infty)$ into $(0,1]$. Next, let $t \in[0, \infty) . D(t, t)=[\varphi(t)]^{-1} \varphi(t)=1$. Hence, for fixed $t \in[0, \infty), r \longmapsto D(r, t)$ maps $[0, t]$ into $(0,1]$. Thus Definition 2 (ii) also holds. Hence, $D$ is a discount function. If, in addition, $\varphi(0)=1$, then $D(0, t)=[\varphi(0)]^{-1} \varphi(t)=\varphi(t)$.

Now suppose that $\varphi$ is onto. Let $p \in(0,1]$. Hence, also, $\varphi(r) p \in(0,1]$. Since $\varphi$ is onto $(0,1]$, we get $\varphi(t)=\varphi(r) p$ for some $t \in[0, \infty)$. But $\varphi(t)=\varphi(r) p \leq \varphi(r)$. Hence, $t \geq r$. We also have $D(r, t)=[\varphi(r)]^{-1} \varphi(t)=[\varphi(r)]^{-1} \varphi(r) p=p$. Hence, for each $r \in[0, \infty)$, $t \longmapsto D(r, t)$ maps $[r, \infty)$ onto $(0,1]$. Thus Definition 2(i) holds with 'into' replaced by 'onto'. Hence, $D$ is a continuous discount function. Since $\varphi:[0, \infty) \stackrel{\text { onto }}{\longrightarrow}(0,1]$ is strictly decreasing, we must have $\varphi(0)=1$. Hence, $D(0, t)=[\varphi(0)]^{-1} \varphi(t)=\varphi(t)$.

For all $r, s$ and $t, D(r, s) D(s, t)=[\varphi(r)]^{-1} \varphi(s)[\varphi(s)]^{-1} \varphi(t)=[\varphi(r)]^{-1} \varphi(t)=$ $D(r, t)$. Hence, $D$ is additive.

(b) Suppose that $D$ is an additive discount function. Then, for all $r, s, t$, where 
$0 \leq r \leq s \leq t, D(r, s) D(s, t)=D(r, t)$. From this, it follows that, for any $s$ and any $t$ $(0 \leq s \leq t): \frac{D(r, t)}{D(r, s)}=D(s, t)$, which is independent of $r$, for all $r \in[0, s]$. Similarly, for any $r$ and any $s(0 \leq r \leq s): \frac{D(r, t)}{D(s, t)}=D(r, s)$, which is independent of $t$, for all $t \in[s, \infty)$. This can only hold if $D(r, t)=F(r) \Phi(t)$, for all $r$ and $t(0 \leq r \leq t)$. In particular, $F(r) \Phi(r)=$ $D(r, r)=1$. Hence, $F(r)=[\Phi(r)]^{-1}$. Hence, $D(r, t)=[\Phi(r)]^{-1} \Phi(t)$. Set $\varphi(t)=$ $\Phi(t) / \Phi(0)$. Then $D(r, t)=[\varphi(r)]^{-1} \varphi(t)$. In particular, $D(0, t)=[\varphi(0)]^{-1} \varphi(t)=\varphi(t)$. Hence, $\varphi$ is a strictly decreasing function from $[0, \infty)$ into $(0,1]$. If $D$ is continuous, so that $D(0, t)$ is onto, then $\varphi$ is also onto.

Proof of Proposition 6: Exponential: $\varphi(t)=e^{-\beta t}$. PPL: $\varphi(0)=1$ and $\varphi(t)=$ $e^{-\delta-\beta t}$ for $t>0$. LP: $\varphi(t)=(1+\alpha t)^{-\frac{\beta}{\alpha}}$. Generalized RS: $\varphi(t)=e^{-Q[w(t)]}$.

Proof of Proposition 7 (Invariance to the choice of reference time):

$$
\begin{gathered}
\left(\left(x_{1}, s_{1}\right),\left(x_{2}, s_{2}\right), \ldots,\left(x_{m}, s_{m}\right)\right) \preceq_{w_{0}, r}\left(\left(y_{1}, t_{1}\right),\left(y_{2}, t_{2}\right), \ldots,\left(y_{n}, t_{n}\right)\right), \\
\Leftrightarrow V_{r}\left(\left(x_{1}, s_{1}\right),\left(x_{2}, s_{2}\right), \ldots,\left(x_{m}, s_{m}\right)\right) \leq V_{r}\left(\left(y_{1}, t_{1}\right),\left(y_{2}, t_{2}\right), \ldots,\left(y_{n}, t_{n}\right)\right), \\
\Leftrightarrow D(0, r) V_{r}\left(\left(x_{1}, s_{1}\right),\left(x_{2}, s_{2}\right), \ldots,\left(x_{m}, s_{m}\right)\right) \leq D(0, r) V_{r}\left(\left(y_{1}, t_{1}\right),\left(y_{2}, t_{2}\right), \ldots,\left(y_{n}, t_{n}\right)\right), \\
\Leftrightarrow D(0, r) \Sigma_{i=1}^{m} v\left(x_{i}\right) D\left(r, s_{i}\right) \leq D(0, r) \Sigma_{i=1}^{n} v\left(y_{i}\right) D\left(r, t_{i}\right), \\
\Leftrightarrow \Sigma_{i=1}^{m} v\left(x_{i}\right) D(0, r) D\left(r, s_{i}\right) \leq \Sigma_{i=1}^{n} v\left(y_{i}\right) D(0, r) D\left(r, t_{i}\right), \\
\Leftrightarrow \Sigma_{i=1}^{m} v\left(x_{i}\right) D\left(0, s_{i}\right) \leq \Sigma_{i=1}^{n} v\left(y_{i}\right) D\left(0, t_{i}\right), \text { by additivity, } \\
\Leftrightarrow V_{0}\left(\left(x_{1}, s_{1}\right),\left(x_{2}, s_{2}\right), \ldots,\left(x_{m}, s_{m}\right)\right) \leq V_{0}\left(\left(y_{1}, t_{1}\right),\left(y_{2}, t_{2}\right), \ldots,\left(y_{n}, t_{n}\right)\right), \\
\Leftrightarrow\left(\left(x_{1}, s_{1}\right),\left(x_{2}, s_{2}\right), \ldots,\left(x_{m}, s_{m}\right)\right) \preceq w_{0}, 0 \\
\end{gathered}
$$

To facilitate the proof of Propositions 8, below, and 22, later, we first establish Lemmas 1 and 2.

Lemma 1 : Let $x \geq 0$ and $y \geq 0$. Then:

(a) $\rho \geq 1 \Rightarrow x^{\rho}+y^{\rho} \leq(x+y)^{\rho}$.

(b) $0<\rho \leq 1 \Rightarrow x^{\rho}+y^{\rho} \geq(x+y)^{\rho}$.

Proof of Lemma 1: Clearly, the results hold for $x=0$. Suppose $x>0$. Let $z=\frac{y}{x}$ and $f(z)=(1+z)^{\rho}-1-z^{\rho}$. Then $f(z)=0$ and $f^{\prime}(z)=\rho\left[(1+z)^{\rho-1}-z^{\rho-1}\right]$, for $z>0$. Suppose $\rho \geq 1$. Then $f^{\prime}(z) \geq 0$. Since $f$ is continuous, it follows that $f(z) \geq 0$ for $z \geq 0$. Part (a) follows from this. Now suppose $0<\rho \leq 1$. Then $f^{\prime}(z) \leq 0$. Since $f$ is continuous, it follows that $f(z) \leq 0$ for $z \geq 0$. Part (b) follows from this.

Lemma 2: Let $\tau>0,0 \leq s<t$ and $r>0$. Let $f(r)=(t+r)^{\tau}-(s+r)^{\tau}-\left(t^{\tau}-s^{\tau}\right)$. Then:

(a) $\tau>1 \Rightarrow f(r)>0$.

(b) $0<\tau<1 \Rightarrow f(r)<0$. 
Proof of Lemma 2: Clearly, $f(0)=0$. Also, $f^{\prime}(r)=\tau\left[(t+r)^{\tau-1}-(s+r)^{\tau-1}\right]$. If $\tau>1$, then $f^{\prime}(r)>0$ for $r>0$. Since $f$ is continuous, it follows that $f(r)>0$ for $r>0$. This establishes part (a). If $0<\tau<1$, then $f^{\prime}(r)<0$ for $r>0$. Since $f$ is continuous, it follows that $f(r)<0$ for $r>0$. This establishes part (b).

Proof of Proposition 8: (a) Suppose $0<\rho \leq 1$. Let $0 \leq r<s<t$. From (2.15), we get:

$$
\begin{aligned}
& D(r, s)=\left[1+\alpha\left(s^{\tau}-r^{\tau}\right)^{\rho}\right]^{-\frac{\beta}{\alpha}} \\
& D(s, t)=\left[1+\alpha\left(t^{\tau}-s^{\tau}\right)^{\rho}\right]^{-\frac{\beta}{\alpha}} \\
& D(r, t)=\left[1+\alpha\left(t^{\tau}-r^{\tau}\right)^{\rho}\right]^{-\frac{\beta}{\alpha}}
\end{aligned}
$$

and, hence,

$$
\begin{gathered}
D(r, s) D(s, t)=\left\{1+\alpha\left[\left(s^{\tau}-r^{\tau}\right)^{\rho}+\left(t^{\tau}-s^{\tau}\right)^{\rho}\right]+\alpha^{2}\left(s^{\tau}-r^{\tau}\right)^{\rho}\left(t^{\tau}-s^{\tau}\right)^{\rho}\right\}^{-\frac{\beta}{\alpha}} \\
<\left\{1+\alpha\left[\left(s^{\tau}-r^{\tau}\right)^{\rho}+\left(t^{\tau}-s^{\tau}\right)^{\rho}\right]\right\}^{-\frac{\beta}{\alpha}}, \text { since } \alpha^{2}\left(s^{\tau}-r^{\tau}\right)^{\rho}\left(t^{\tau}-s^{\tau}\right)^{\rho}>0 \text { and }-\frac{\beta}{\alpha}<0 \\
\leq\left[1+\alpha\left(s^{\tau}-r^{\tau}+t^{\tau}-s^{\tau}\right)^{\rho}\right]^{-\frac{\beta}{\alpha}}, \text { by Lemma 1b and since }-\frac{\beta}{\alpha}<0, \\
=\left[1+\alpha\left(t^{\tau}-r^{\tau}\right)^{\rho}\right]^{-\frac{\beta}{\alpha}} \\
=D(r, t) .
\end{gathered}
$$

(b) It is sufficient to give an example. Let $\alpha=\tau=1$ and $\rho=2$. Hence, $D(0,1) D(1,2)=$ $4^{-\beta}>5^{-\beta}=D(0,2)$. Hence, for $\alpha=\tau=1$ and $\rho=2, D$ cannot be additive or subadditive. However, for the same parameter values, we have $D(0,10) D(10,20)=10201^{-\beta}<$ $401^{-\beta}=D(0,20)$. Hence, $D$ cannot be supper additive either. ${ }^{34}$

(c) Let $0 \leq s<t$. (ii) is obvious from inspecting (2.15). Let $r>0$. (iii) For $\tau>1$, Lemma 2 (a) gives $D(s+r, t+r)=\left\{1+\alpha\left[(t+r)^{\tau}-(s+r)^{\tau}\right]^{\rho}\right\}^{-\frac{\beta}{\alpha}}<\left\{1+\alpha\left[t^{\tau}-s^{\tau}\right]^{\rho}\right\}^{-\frac{\beta}{\alpha}}$ $=D(s, t)$. (i) For $0<\tau<1$, Lemma 2(b) gives $D(s+r, t+r)=\left\{1+\alpha\left[(t+r)^{\tau}-(s+r)^{\tau}\right]^{\rho}\right\}^{-\frac{\beta}{\alpha}}$ $>\left\{1+\alpha\left[t^{\tau}-s^{\tau}\right]^{\rho}\right\}^{-\frac{\beta}{\alpha}}=D(s, t)$.

Proof of Proposition 9 (Properties of a delay function): Let $D$ be a discount function and $\Psi$ and $\Phi$ two corresponding delay functions. Let $s, t \in[0, \infty)$. Then $D(0, \Phi(s, t))=D(0, s) D(0, t)=D(0, \Psi(s, t))$. Since $D(0, r)$ is strictly decreasing in $r$, we must have $\Phi(s, t)=\Psi(s, t)$. This establishes (a). Using Definition 2, it is straightforward to check that properties (b) to (d) follow from Definition 7. Now, suppose $v(x)$ $=v(y) D(0, t)$. Multiply both sides by $D(0, s)$ to get $v(x) D(0, s)=v(y) D(0, s) D(0, t)$ $=v(y) D(0, \Psi(s, t))$. Conversely, suppose $v(x) D(0, s)=v(y) D(0, \Psi(s, t))$. Then $v(x) D(0, s)=v(y) D(0, s) D(0, t)$. Since $D(0, s)>0$, we can cancel it to get $v(x)$ $=v(y) D(0, t)$. This establishes (e) and completes the proof.

\footnotetext{
${ }^{34}$ Other examples can be given to show that there is nothing special about $r=0, \alpha=1, \tau=1$, or $\rho=2$, as long as $\rho>1$.
} 
Proof of Proposition 10 (Existence of a delay function): Let $D$ be a continuous discount function. Let $s, t \in[0, \infty)$. Then $D(0, s), D(0, t) \in(0,1]$. Hence also $D(0, s) D(0, t) \in(0,1]$. Since $r \mapsto D(0, r)$ is onto $(0,1]$, there is some $T \in[0, \infty)$ such that $D(0, s) D(0, t)=D(0, T)$. Since $D(0, r)$ is strictly decreasing in $r$, this $T$ is unique. Set $T=\Psi(s, t)$. The function, $\Psi(s, t)$, thus defined, is a delay function corresponding to $D$.

Proof of Proposition 11: (Representation Theorem 1) Let $\alpha>0$ and $\beta>0$. It is easy to verify that $D(0, t)=[1+\alpha \psi(t)]^{-\frac{\beta}{\alpha}}$ is an $(\alpha, \beta)$-representation of the discount function $D(r, t)$ if, and only if, $\psi(t)=\frac{1}{\alpha}[D(0, t)]^{-\frac{\alpha}{\beta}}-1$. From this, using Definition 2 , it is straightforward to verify that part (a) holds. It follows that the inverse of $\psi$ exists and $\psi^{-1}:[0, \infty) \stackrel{\text { onto }}{\longrightarrow}[0, \infty)$ is strictly increasing with $\psi(0)=0$, this establishes part (b). We now turn to part (c). We have $[1+\alpha \psi(\Psi(s, t))]^{-\frac{\beta}{\alpha}}=D(0, \Psi(s, t))=$ $D(0, s) D(0, t)=[1+\alpha \psi(s)]^{-\frac{\beta}{\alpha}}[1+\alpha \psi(t)]^{-\frac{\beta}{\alpha}}=\left[1+\alpha \psi(s)+\alpha \psi(t)+\alpha^{2} \psi(s) \psi(t)\right]^{-\frac{\beta}{\alpha}}$. Hence, $[1+\alpha \psi(\Psi(s, t))]^{-\frac{\beta}{\alpha}}=\left[1+\alpha \psi(s)+\alpha \psi(t)+\alpha^{2} \psi(s) \psi(t)\right]^{-\frac{\beta}{\alpha}}$. From the latter it follows that $\psi(\Psi(s, t))=\psi(s)+\psi(t)+\alpha \psi(s) \psi(t)$. Hence, $\Psi(s, t)=\psi^{-1}[\psi(s)+\psi(t)+\alpha \psi(s) \psi(t)]$.

Proof of Proposition 12 (Representation Theorem 2): Let

$$
\psi:[0, \infty) \stackrel{\text { onto }}{\rightarrow}[0, \infty) \text { be strictly increasing (hence, } \psi(0)=0)
$$

and let

$$
\Psi(s, t)=\psi^{-1}(\psi(s)+\psi(t)+\alpha \psi(s) \psi(t)), \alpha>0 .
$$

Let $D$ be a continuous discount function with delay function, $\Psi$. Then

$$
D(0, s) D(0, t)=D(0, \Psi(s, t)), s \geq 0, t \geq 0 .
$$

From (7.2) and (7.3), we get

$$
D(0, s) D(0, t)=D\left(0, \psi^{-1}(\psi(s)+\psi(t)+\alpha \psi(s) \psi(t))\right), s \geq 0, t \geq 0 .
$$

From $(7.1)$, we get that $\psi^{-1}$ exists and $\psi^{-1}:[0, \infty) \stackrel{\text { onto }}{\rightarrow}[0, \infty)$ is strictly increasing with $\psi^{-1}(0)=0$. Bear these facts in mind for when the functions $G$ and $h$ are defined, below $((7.7)$ and $(7.10))$.

Let

$$
X=1+\alpha \psi(s), Y=1+\alpha \psi(t) .
$$

Hence

$$
\begin{gathered}
\psi(s)=\frac{X-1}{\alpha}, \psi(t)=\frac{Y-1}{\alpha}, s=\psi^{-1}\left(\frac{X-1}{\alpha}\right), t=\psi^{-1}\left(\frac{Y-1}{\alpha}\right), \\
\psi(s)+\psi(t)+\alpha \psi(s) \psi(t)=\frac{X Y-1}{\alpha} \\
\psi^{-1}(\psi(s)+\psi(t)+\alpha \psi(s) \psi(t))=\psi^{-1}\left(\frac{X Y-1}{\alpha}\right) .
\end{gathered}
$$


Define the function $G:[1, \infty) \rightarrow(0, \infty)$ by

$$
G(X)=D\left(0, \psi^{-1}\left(\frac{X-1}{\alpha}\right)\right) .
$$

Hence,

$$
G(Y)=D\left(0, \psi^{-1}\left(\frac{Y-1}{\alpha}\right)\right), G(X Y)=D\left(0, \psi^{-1}\left(\frac{X Y-1}{\alpha}\right)\right) .
$$

From (7.4), (7.6), (7.7), (7.8)

$$
\begin{aligned}
G(X Y) & =D\left(0, \psi^{-1}\left(\frac{X Y-1}{\alpha}\right)\right) \\
& =D\left(0, \psi^{-1}(\psi(s)+\psi(t)+\alpha \psi(s) \psi(t))\right) \\
& =D(0, s) D(0, t) \\
& =D\left(0, \psi^{-1}\left(\frac{X-1}{\alpha}\right)\right) D\left(0, \psi^{-1}\left(\frac{Y-1}{\alpha}\right)\right) \\
& =G(X) G(Y) .
\end{aligned}
$$

Define $^{35}$ the function $h:[0, \infty) \rightarrow(0, \infty)$ by

$$
h(y)=G\left(e^{y}\right), y \geq 0
$$

Hence, and in the light of Definition 2(i), $h$ satisfies $^{36}$ :

$$
h:[0, \infty) \rightarrow(0, \infty) \text { is strictly decreasing and } h(x+y)=h(x) h(y)
$$

As is well known, see for example Corollary 1.4.11 in Eichhorn (1978) or Theorem 1, page 38, of Aczel (1966), the unique solution to (7.11) is the exponential function

$$
h(y)=e^{c y}, y \geq 0, c<0
$$

(7.5), (7.6), (7.8), (7.10), (7.12) give, in succession,

$$
\begin{gathered}
h(y)=\left(e^{y}\right)^{c} \\
G\left(e^{y}\right)=\left(e^{y}\right)^{c} \\
G(Y)=Y^{c}
\end{gathered}
$$

\footnotetext{
${ }^{35}$ It is tempting, at this stage, to take a shortcut and conclude, from the fact that $G(X Y)=G(X) G(Y)$, that, necessarily, $G(X)=X^{c}$. However, the relevant theorem (Theorem 1.9.13 in Eichhorn (1978) or Theorem 3, page 41, in Aczel (1966)) requires that $G(X)$ be defined for all $X>0$. However, $\psi^{-1}(t)$ is not defined for $t<0$ and, hence, $G(X)$ is not defined for $X<1$.

${ }^{36} \mathrm{It}$ is sufficient that $h$ be strictly decreasing in some interval: $(a, a+\delta), a \geq 0, \delta>0$.
} 


$$
\begin{gathered}
D\left(0, \psi^{-1}\left(\frac{Y-1}{\alpha}\right)\right)=Y^{c} \\
D\left(0, \psi^{-1}(\psi(t))\right)=[1+\alpha \psi(t)]^{c} \\
D(0, t)=[1+\alpha \psi(t)]^{c} .
\end{gathered}
$$

Let

$$
\beta=-\alpha c
$$

(7.13), (7.14) give

$$
D(0, t)=[1+\alpha \psi(t)]^{-\frac{\beta}{\alpha}}, \alpha>0, \beta>0, t \geq 0
$$

where $\beta>0$ because $\alpha>0$ and $c<0$.

Proof of Proposition 13 (Extension Theorem 1): (a) Let $f$ and $g$ be extension functions corresponding to the discount function $D$. Let $r \in[0, \infty)$ and $t \in[r, \infty)$. Then, by Definition 11(ii), $D(0, f(r, t))=D(r, t)=D(0, g(r, t))$. Since $D(r, s)$ is strictly decreasing in $s$, it follows that $f(r, t)=g(r, t)$. Hence, $f=g$. (b) We have $D(0, t)=$ $D(0, f(0, t))$. Since $D(0, s)$ is strictly decreasing in $s$, it follows that $f(0, t)=t$. We also have $D(0, f(r, r))=D(r, r)=1=D(0,0)$, i.e., $D(0, f(r, r))=D(0,0)$. Again, since $D(0, t)$ is strictly decreasing in $t$, it follows that $f(r, r)=0$. Since $D(0, f(r, t))$ $=D(r, t)$ and $D(r, t)$ is strictly decreasing in $t$, it follows that $f(r, t)$ must be strictly increasing in $t$. Since $D(r, t)$ is strictly increasing in $r$, it follows that $f(r, t)$ must be strictly decreasing in $r$. This completes the proof that $f$ is an extension function. (c) Since $D(r, t)=D(0, f(r, t))$, it follows that $v(x)=v(y) D(r, t)$ if, and only if, $v(x)=$ $v(y) D(0, f(r, t))$.

Proof of Proposition 14 (Extension Theorem 2): Let $r \in[0, \infty)$ and $t \in[r, \infty)$. Then $D(r, t) \in(0,1]$. Since $D$ is a continuous discount function, it follows that $s \longmapsto D(0, s)$ is onto $(0,1]$. Hence, for some $T \in(0,1], D(r, t)=D(0, T)$. Since $D(r, s)$ is strictly decreasing in $s$, it follows that this $T$ is unique. Set $f(r, t)=T$. The function, $f$, thus defined has the property $D(r, t)=D(0, f(r, t))$. Hence, $f$ is an extension function corresponding to $D$. Let $r \in[0, \infty)$. Let $t \in[0, \infty)$. Then $D(0, t) \in(0,1]$. Since, $D$ is a continuous discount function, it maps $[r, \infty)$ onto $(0,1]$. Hence $D(r, s)=D(0, t)$, for some $s \in[r, \infty)$. But since $f$ is an extension function corresponding to $D$, we have $D(r, s)=$ $D(0, f(r, s))$. Hence, $D(0, f(r, s))=D(0, t)$. Since $D(0, q)$ is strictly decreasing in $q$, it follows that $f(r, s)=t$. Hence, $t \longmapsto f(r, t)$ maps $[r, \infty)$ onto $[0, \infty)$.

Proof of Proposition 15 (Extension Theorem 3): (a) Since $f: \Delta \rightarrow[0, \infty)$ and $\varphi:[0, \infty) \rightarrow(0,1]$, it follows that $\varphi \circ f: \Delta \rightarrow(0,1]$. Let $r \in[0, \infty)$. Then $t \mapsto \varphi(f(r, t))$ maps $[r, \infty)$ into $(0,1]$ and is strictly decreasing with $D(r, r)=\varphi(f(r, r))=\varphi(0)=1$. Let $t \in[0, \infty)$. Then $r \mapsto \varphi(f(r, t))$ maps $[0, t]$ into $(0,1]$ and is strictly decreasing. 
Thus, $D(r, t)=\varphi(f(r, t))$ satisfies all parts of Definition 2 and, hence, is a discount function. (b) $D(0, f(r, t))=\varphi(f(0, f(r, t)))=\varphi(f(r, t))=D(r, t)$. Hence, $f$ is the extension function corresponding to $D$ and $D(r, t)$ is the $f$-extension of $D(0, t)$. (c) Let $r \in[0, \infty)$. Let $p \in(0,1]$. Since $\varphi$ is onto, there exists $t \in[0, \infty)$ such that $p=\varphi(t)$. Since $s \mapsto f(r, s)$ maps $[r, \infty)$ onto $[0, \infty)$, there exists $s \in[r, \infty)$ such that $f(r, s)=t$. Hence, $D(r, s)=\varphi(f(r, s))=\varphi(t)=p$. Hence, $s \mapsto D(r, s)$ maps $[r, \infty)$ onto $[0, \infty)$. Hence, $D$ is a continuous discount function.

Proof of Proposition 16 (Characterization Theorem 1): Let $D$ be a continuous discount function. Then, by Representation Theorem 1 (Proposition 11) and Extension Theorem 2 (Proposition 14) $D(r, t)=D(0, f(r, t))=[1+\alpha \psi(f(r, t))]^{-\frac{\beta}{\alpha}}$, where $\alpha>0$, $\beta>0, \psi$ is a continuous seed function and $f$ is a continuous extension function. Conversely, if $D(r, t)=[1+\alpha \psi(f(r, t))]^{-\frac{\beta}{\alpha}}$, where $\alpha>0, \beta>0, \psi$ is a continuous seed function and $f$ is a continuous extension function, then $D$ satisfies all the conditions of Definition 2, with 'onto' replacing 'into' in part (i). Hence, $D$ is a continuous discount function. Uniqueness of $f$ follows from Extension Theorem 1 (Proposition 13).

Proof of Proposition 17 (Characterization Theorem 2): Follows from Definition 6 and Characterization Theorem 1 (Proposition 16).

Proof of Proposition 18 (Characterization Theorem 3): Let $f(r, t)=\psi^{-1}\left(\frac{\psi(t)-\psi(r)}{1+\alpha \psi(r)}\right)$. Then $D(r, t)=[1+\alpha \psi(f(r, t))]^{-\frac{\beta}{\alpha}}=\left[1+\alpha \psi\left(\psi^{-1}\left(\frac{\psi(t)-\psi(r)}{1+\alpha \psi(r)}\right)\right)\right]^{-\frac{\beta}{\alpha}}=\left[1+\alpha \frac{\psi(t)-\psi(r)}{1+\alpha \psi(r)}\right]^{-\frac{\beta}{\alpha}}$ $=\left[\frac{1+\alpha \psi(t)}{1+\alpha \psi(r)}\right]^{-\frac{\beta}{\alpha}}$, which is additive, by Proposition 5 (aiii). Conversely, suppose $D$ is additive. Then, by Proposition $5(\mathrm{~b}), D(r, t)=[\varphi(r)]^{-1} \varphi(t)$ for some strictly decreasing real valued function, $\varphi:[0, \infty) \stackrel{\text { onto }}{\rightarrow}(0,1], \varphi(0)=1$. Let $D(r, t)=[1+\alpha \psi(f(r, t))]^{-\frac{\beta}{\alpha}}$. Then $\varphi(t)=D(0, t)=[1+\alpha \psi(t)]^{-\frac{\beta}{\alpha}}$. Hence, $[1+\alpha \psi(f(r, t))]^{-\frac{\beta}{\alpha}}=\left[\frac{1+\alpha \psi(t)}{1+\alpha \psi(r)}\right]^{-\frac{\beta}{\alpha}}$ and, hence, $1+\alpha \psi(f(r, t))=\frac{1+\alpha \psi(t)}{1+\alpha \psi(r)}$. From which it follows that $f(r, t)=\psi^{-1}\left(\frac{\psi(t)-\psi(r)}{1+\alpha \psi(r)}\right)$.

Proof of Proposition 19: All the claims can be verified by straightforward calculations. However, when dealing with PPL, do not use Proposition 11, as PPL is not continuous. So, for example, instead of using part b of Proposition 11, check directly that $D(0, s) D(0, t)=D(0, \Psi(s, t))$.

Proof of Proposition 20 (Representation Theorem 3): Since $\psi(0)=0$ and $\psi$ is strictly increasing (Proposition 11a), it follows that $\psi(t)>0 \Leftrightarrow t>0$. Let $s>0$ and $t>0$, then $D(0, s) D(0, t)<D(0, s+t) \Leftrightarrow[1+\alpha \psi(s)]^{-\frac{\beta}{\alpha}}[1+\alpha \psi(t)]^{-\frac{\beta}{\alpha}}<[1+\alpha \psi(s+t)]^{-\frac{\beta}{\alpha}}$ $\Leftrightarrow[1+\alpha \psi(s)][1+\alpha \psi(t)]>1+\alpha \psi(s+t) \Leftrightarrow \psi(s+t)<\psi(s)+\psi(t)+\alpha \psi(s) \psi(t)$ $\Leftrightarrow s+t<\psi^{-1}[\psi(s)+\psi(t)+\alpha \psi(s) \psi(t)] \Leftrightarrow s+t<\Psi(s, t)$. From this chain it follows that $(\mathrm{c}) \Leftrightarrow(\mathrm{a}) \Leftrightarrow(\mathrm{b})$.

Proof of Proposition 21 (Characterization Theorem 4): Assume $\psi_{+}$is $\alpha_{+}$-subadditive. Let $0<x<y, v(x)=v(y) D_{+}(0, t)$ and $s>0$. Then $0<v(x)<v(y)$. It follows 
that $D_{+}(0, t)<1$ and, hence, $t>0$. From Representation Theorem 3(c) (Proposition $20)$, it follows that $D_{+}(0, s) D_{+}(0, t)<D_{+}(0, s+t)$. Hence, $v(y) D_{+}(0, t) D_{+}(0, s)<$ $v(y) D_{+}(0, s+t)$. Hence, $v(x) D_{+}(0, s)<v(y) D_{+}(0, s+t)$. Hence, the common difference effect holds for gains.

Conversely, assume that the common difference effect holds for gains. Let $s>0$ and $t>0$. Hence, $0<s<s+t$. Hence, by Proposition 2.10, for some $x, 0 \leq x \leq 1$, $v(x)=v(1) D_{+}(0, t)$. Hence, $x>0$. Hence, $v(x) D_{+}(0, s)<v(1) D_{+}(0, s+t)$. Hence, $v(1) D_{+}(0, s) D_{+}(0, t)<v(1) D_{+}(0, s+t)$. Hence, $D_{+}(0, s) D_{+}(0, t)<D_{+}(0, s+t)$. From Representation Theorem 3 (Proposition 20), it follows that $\psi_{+}$is $\alpha_{+}$-subadditive.

Hence, preferences exhibit the common difference effect for gains if, and only if, the seed function for gains, $\psi_{+}$, is $\alpha_{+}$-subadditive. A similar argument shows that preferences exhibit the common difference effect for losses if, and only if, the seed function for losses, $\psi_{-}$, is $\alpha_{-}$-subadditive.

Proof of Proposition 22: Let $\psi_{+}(t)=t^{\gamma_{+}}$, where $0<\gamma_{+} \leq 1$. Then, for $s>0$ and $t>0, \psi_{+}(s+t)=(s+t)^{\gamma_{+}} \leq t^{\gamma_{+}}+s^{\gamma_{+}}<s^{\gamma_{+}}+t^{\gamma_{+}}+\alpha_{+} s^{\gamma_{+}} t^{\gamma_{+}}=\psi_{+}(s)+\psi_{+}(t)+$ $\alpha_{+} \psi_{+}(s) \psi_{+}(t)$. Hence, by Characterization Theorem 4 (Proposition 21), preferences that exhibit $\gamma$-delay (Definition 13), also exhibit the common difference effect for gains. Similarly, they exhibit the common difference effect for losses.

Proof of Proposition 23: Let preferences with continuous discount function $D_{+}$ (gains) and $D_{-}$(losses) exhibit $\gamma$-delay. Then, by Definition 13, their respective delay functions are $\Psi_{+}(s, t)=\left(s^{\gamma_{+}}+t^{\gamma_{+}}+\alpha_{+} s^{\gamma_{+}} t^{\gamma_{+}}\right)^{\frac{1}{\gamma_{+}}}, \alpha_{+}>0,0<\gamma_{+} \leq 1$ and $\Psi_{-}(s, t)=$ $\left(s^{\gamma_{-}}+t^{\gamma_{-}}+\alpha_{-} s^{\gamma_{-}} t^{\gamma_{-}}\right)^{\frac{1}{\gamma_{-}}}, \alpha_{-}>0,0<\gamma_{-} \leq 1$. Hence, by Representation Theorem 2 (Proposition 12), we must have $D_{+}(0, t)=\left(1+\alpha_{+} t^{\gamma_{+}}\right)^{-\frac{\beta_{+}}{\alpha_{+}}}$, for some $\beta_{+}>0$, and $D_{-}(0, t)=\left(1+\alpha_{-} t^{\gamma_{-}}\right)^{-\frac{\beta_{-}}{\alpha_{-}}}$, for some $\beta_{-}<0$.

Proof of Proposition 24: By Proposition 23 we have, necessarily, $D_{+}(0, t)=$ $\left(1+\alpha_{+} t^{\gamma_{+}}\right)^{-\frac{\beta_{+}}{\alpha_{+}}}$, for some $\beta_{+}>0$, and $D_{-}(0, t)=\left(1+\alpha_{-} t^{\gamma_{-}}\right)^{-\frac{\beta_{-}}{\alpha_{-}}}$, for some $\beta_{-}<$ 0 . Hence, by Characterization Theorem 3 (Proposition 18) we must have $D_{+}(r, t)=$ $\left(\frac{1+\alpha_{+} t^{\gamma+}}{1+\alpha_{+} r^{\gamma+}}\right)^{-\frac{\beta_{+}}{\alpha_{+}}}$and $D_{-}(r, t)=\left(\frac{1+\alpha_{-} t^{\gamma_{-}}}{1+\alpha_{-} r_{-}}\right)^{-\frac{\beta_{-}}{\alpha_{-}}}$.

Proof of Proposition 25: By Proposition 23 we have, $D_{+}(0, t)=\left(1+\alpha_{+} t^{\gamma_{+}}\right)^{-\frac{\beta_{+}}{\alpha_{+}}}$, for some $\beta_{+}>0$, and $D_{-}(0, t)=\left(1+\alpha_{-} t^{\gamma_{-}}\right)^{-\frac{\beta_{-}}{\alpha_{-}}}$, for some $\beta_{-}<0$. Hence, $D_{+}(r, t)=$ $D_{+}\left(0, f_{+}(r, t)\right)=\left[1+\alpha_{+}\left[f_{+}(r, t)\right]^{\gamma_{+}}\right]^{-\frac{\beta_{+}}{\alpha_{+}}}=\left[1+\alpha_{+}\left[\left(t^{\tau_{+}}-r^{\tau_{+}}\right)^{\frac{1}{\tau_{+}}}\right]^{\gamma_{+}}\right]^{-\frac{\beta_{+}}{\alpha_{+}}}=\left[1+\alpha_{+}\left(t^{\tau_{+}}-r^{\tau_{+}}\right)^{\frac{\gamma_{+}}{\tau_{+}}}\right]^{-\frac{\beta_{+}}{\alpha_{+}}}$ $=\left[1+\alpha_{+}\left(t^{\tau_{+}}-r^{\tau_{+}}\right)^{\rho_{+}}\right]^{-\frac{\beta_{+}}{\alpha_{+}}}$. Similarly, $D_{-}(r, t)=\left[1+\alpha_{-}\left(t^{\tau_{-}}-r^{\tau_{-}}\right)^{\rho_{-}}\right]^{-\frac{\beta_{-}}{\alpha_{-}}}$.

To facilitate what follows, we define two functions, $\widetilde{v}_{+}$and $\widetilde{v}_{-}$, associated with the value function (2.6), 


\section{Definition 15 :}

$$
\begin{aligned}
& \widetilde{v}_{+}(x)=\ln v\left(e^{x}\right),-\infty<x<\infty, \\
& \widetilde{v}_{-}(x)=\ln \left(-v\left(-e^{x}\right)\right),-\infty<x<\infty .
\end{aligned}
$$

Proof of Proposition 26: Suppose $0<x<y$. By Proposition 3, $v(x)=v(y) D_{+}(0, t)$ for some $t \in[0, \infty)$. Hence, $\frac{v(x)}{v(y)}=D_{+}(0, t)$. By assumption A1, $v(-x)>v(-y) D_{-}(0, t)$. Since $-y<0$, it follows that $v(-y)<0$ and, hence, $\frac{v(-x)}{v(-y)}<D_{-}(0, t)$. Then, $D_{+}(0, t)=$ $D_{-}(0, t)$ gives $\frac{v(-x)}{v(-y)}<\frac{v(x)}{v(y)}$. Taking logs gives $\ln v(y)-\ln v(x)<\ln (-v(-y))-\ln (-v(-x))$ and, hence, $\frac{\ln v(y)-\ln v(x)}{\ln y-\ln x}<\frac{\ln (-v(-y))-\ln (-v(-x))}{\ln y-\ln x}$. Letting $\widetilde{x}=\ln x$ and $\widetilde{y}=\ln y$, we get $\frac{\widetilde{v}_{+}(\widetilde{y})-\widetilde{v}_{+}(\widetilde{x})}{\widetilde{y}-\widetilde{x}}<\frac{\widetilde{v}_{-}(\widetilde{y})-\widetilde{v}_{-}(\widetilde{x})}{\widetilde{y}-\widetilde{x}}$. Take limits as $\widetilde{y} \rightarrow \widetilde{x}$, to get $\frac{d \widetilde{v}_{+}}{d \widetilde{x}} \leq \frac{d \widetilde{v}_{-}}{d \widetilde{x}}$, from which it follows that $\epsilon_{v}(x) \leq \epsilon_{v}(-x)$.

Proof of Proposition 27: Let

$$
0<x<y(\text { or } y<x<0)
$$

By Proposition 3, there is a time, $t$, such that the consumer is indifferent between receiving the increment $x$ now and receiving the increment $y, t$-periods from now. Then, letting $v$ be the value function and $D_{ \pm}$the discount function, we get

$$
v(x)=v(y) D_{+}(0, t)\left(v(x)=v(y) D_{-}(0, t)\right) .
$$

Let

$$
a>1
$$

then the magnitude effect, assumption A2, predicts that

$$
v(a x)<v(a y) D_{+}(0, t)\left(v(a x)>v(a y) D_{-}(0, t)\right)
$$

(7.18) gives

$$
\frac{v(x)}{v(y)}=D_{+}(0, t) \quad\left(\frac{v(x)}{v(y)}=D_{-}(0, t)\right) .
$$

Since $y, a$ are positive (respectively, $y$ is negative), it follows that $a y$, and hence, $v(a y)$ are also positive (respectively, negative). Hence, (7.20) gives

$$
\frac{v(a x)}{v(a y)}<D_{+}(0, t) \quad\left(\frac{v(a x)}{v(a y)}>D_{-}(0, t)\right),
$$

(7.21) and (7.22) give ${ }^{37}$

$$
\frac{v(a y)}{v(a x)}>\frac{v(y)}{v(x)}
$$

\footnotetext{
${ }^{37}$ In the course of their proof, LP derive, incorrectly, the formula (LP (18) p583): $\frac{v(x)}{v(y)}<\frac{v(a x)}{v(a y)}, 0<x<$ $y ; a>1$ (the first $<$ should be $>$ )
} 
Start with the case $0<x<y$. Since $a>0, x>0$ and $y>0$, and since $v(0)=0$ and $v$ is strictly increasing, it follows that $v(x), v(y), v(a x)$ and $v(a y)$ are all positive. Hence, we can take their logs. Let $\widetilde{x}=\ln x, \widetilde{y}=\ln y, \widetilde{a}=\ln a$ (hence, $x=e^{\widetilde{x}}, y=e^{\widetilde{y}}, a=e^{\widetilde{a}}$ ). Then, since $x<y$ and $a>1$, it follows that $\widetilde{x}<\widetilde{y}, \widetilde{a}>0$. Let $\widetilde{v}(\widetilde{x})=\ln v\left(e^{\widetilde{x}}\right)$, then, by taking logs, and recalling that the logarithmic function is strictly increasing and changes multiplication to addition, (7.23) gives:

$$
\begin{gathered}
\ln v(a y)-\ln v(a x)>\ln v(y)-\ln v(x), \\
\ln v\left(e^{\widetilde{a}} e^{\widetilde{y}}\right)-\ln v\left(e^{\widetilde{a}} e^{\widetilde{x}}\right)>\ln v\left(e^{\widetilde{y}}\right)-\ln v\left(e^{\widetilde{x}}\right), \\
\ln v\left(e^{\widetilde{a}+\widetilde{y}}\right)-\ln v\left(e^{\widetilde{a}+\widetilde{x}}\right)>\ln v\left(e^{\widetilde{y}}\right)-\ln v\left(e^{\widetilde{x}}\right) .
\end{gathered}
$$

Since $\widetilde{v}(x)=\ln v\left(e^{x}\right)$, we get

$$
\begin{gathered}
\widetilde{v}(\widetilde{y}+\widetilde{a})-\widetilde{v}(\widetilde{x}+\widetilde{a})>[\widetilde{v}(\widetilde{y})-\widetilde{v}(\widetilde{x})], \\
\widetilde{v}(\widetilde{y}+\widetilde{a})-\widetilde{v}(\widetilde{x}+\widetilde{a})-[\widetilde{v}(\widetilde{y})-\widetilde{v}(\widetilde{x})]>0 .
\end{gathered}
$$

Take $\delta x>0, \widetilde{a}=\delta x, \widetilde{y}=\widetilde{x}+\delta x$, then (7.24) gives:

$$
\begin{aligned}
& \widetilde{v}(\widetilde{x}+2 \delta x)-\widetilde{v}(\widetilde{x}+\delta x)-[\widetilde{v}(\widetilde{x}+\delta x)-\widetilde{v}(\widetilde{x})]>0, \\
& \frac{\widetilde{v}(\widetilde{x}+2 \delta x)-\widetilde{v}(\widetilde{x}+\delta x)-[\widetilde{v}(\widetilde{x}+\delta x)-\widetilde{v}(\widetilde{x})]}{(\delta x)^{2}}>0 .
\end{aligned}
$$

Now

$$
\begin{gathered}
{\left[\frac{d \widetilde{v}}{d x}\right]_{x=\widetilde{x}}=\lim _{\delta x \rightarrow 0} \frac{\widetilde{v}(\widetilde{x}+\delta x)-v(\widetilde{x})}{\delta x},} \\
{\left[\frac{d \widetilde{v}}{d x}\right]_{x=\widetilde{x}+\delta x}=\lim _{\delta x \rightarrow 0} \frac{\widetilde{v}(\widetilde{x}+2 \delta x)-\widetilde{v}(\widetilde{x}+\delta x)}{\delta x}} \\
{\left[\frac{d^{2} \widetilde{v}}{d x^{2}}\right]_{x=\widetilde{x}}=\lim _{\delta x \rightarrow 0} \frac{\left[\frac{d \widetilde{v}}{d x}\right]_{x=\widetilde{x}+\delta x}-\left[\frac{d \widetilde{v}}{d x}\right]_{x=\widetilde{x}}}{\delta x} .}
\end{gathered}
$$

Hence,

$$
\begin{gathered}
{\left[\frac{d^{2} \widetilde{v}}{d x^{2}}\right]_{x=\widetilde{x}}=\lim _{\delta x \rightarrow 0} \frac{\lim _{\delta x \rightarrow 0} \frac{\widetilde{v}(\widetilde{x}+2 \delta x)-\widetilde{v}(\widetilde{x}+\delta x)}{\delta x}-\lim _{\delta x \rightarrow 0} \frac{\widetilde{v}(\widetilde{x}+\delta x)-\widetilde{v}(\widetilde{x})}{\delta x},}{\delta x},} \\
{\left[\frac{d^{2} \widetilde{v}}{d x^{2}}\right]_{x=\widetilde{x}}=\lim _{\delta x \rightarrow 0} \frac{\widetilde{v}(\widetilde{x}+2 \delta x)-\widetilde{v}(\widetilde{x}+\delta x)}{\delta x}-\frac{\widetilde{v}(\widetilde{x}+\delta x)-\widetilde{v}(\widetilde{x})}{\delta x}} \\
{\left[\frac{d^{2} \widetilde{v}}{d x^{2}}\right]_{x=\widetilde{x}}=\lim _{\delta x \rightarrow 0} \frac{\widetilde{v}(\widetilde{x}+2 \delta x)-\widetilde{v}(\widetilde{x}+\delta x)-[\widetilde{v}(\widetilde{x}+\delta x)-\widetilde{v}(\widetilde{x})]}{(\delta x)^{2}} .}
\end{gathered}
$$


Since the limit of a converging sequence of positive numbers in non-negative, we get, from (7.25) and (7.26):

$$
\begin{gathered}
{\left[\frac{d^{2} \widetilde{v}}{d x^{2}}\right]_{x=\widetilde{x}} \geq 0} \\
{\left[\frac{d}{d x}\left(\frac{d}{d x} \widetilde{v}(x)\right)\right]_{x=\widetilde{x}} \geq 0 .} \\
\frac{d}{d x}(\epsilon(x)) \geq 0 .
\end{gathered}
$$

If $\epsilon(x)$ were constant on some non-empty open interval, then the value function would take the form $v(x)=c x^{\gamma}$ on that interval, and subproportionality. (7.23) would be violated. Hence $\epsilon^{\prime}(x)>0$ almost everywhere. Thus $\epsilon(x)$ increases with $x$.

Now consider the case $y<x<0$. Then (7.23) still holds. But now we define $\widetilde{x}=$ $\ln (-x), \widetilde{y}=\ln (-y)$ and $\widetilde{v}(\widetilde{x})=-\ln \left(-v\left(-e^{\widetilde{x}}\right)\right)$. As before, (7.24) holds and $v^{\prime}(x)>0$ almost everywhere. Thus $\epsilon(x)$ increases with $x$.

It then follows that the value function is more elastic for outcomes that are larger in absolute magnitude.

Proof of Proposition 28: That $\epsilon_{v}>0$, follows from (2.6) and (2.7). Also from (2.7) we get:

$$
v^{\prime \prime}(x)=\frac{v(x)}{x}\left[\epsilon_{v}^{\prime}-\frac{\epsilon_{v}\left(1-\epsilon_{v}\right)}{x}\right] .
$$

If $x>0$ then $v(x)>0, v^{\prime \prime}(x)<0, \epsilon_{v}^{\prime}(x) \geq 0$. From (7.27) it follows that, necessarily, $\epsilon_{v}<1$.

If $x<0$ then $v(x)<0, v^{\prime \prime}(x)>0, \epsilon_{v}^{\prime}(x) \leq 0$. From (7.27), it follows that, again, $\epsilon_{v}<1$.

Proof of Proposition 29: Suppose $0<x<y$. By Proposition 3, $v(x)=v(y) D_{+}(0, t)$ for some $t \in[0, \infty)$. By A1, $v(-x)>v(-y) D_{-}(0, t)$. Hence, from A6, $-\lambda v(x)>$ $-\lambda v(y) D_{-}(0, t)$. Hence, $v(y) D_{-}(0, t)>v(x)=v(y) D_{+}(0, t)$. It follows that $D_{-}(0, t)>$ $D_{+}(0, t)$. We also get $\frac{v(-x)}{v(-y)}=\frac{-\lambda v(x)}{-\lambda v(y)}=\frac{v(x)}{v(y)}$. Finally, $\epsilon_{v}(-x)=\frac{-x}{v(-x)} v^{\prime}(-x)=\frac{-x}{-\lambda v(x)}\left(-\frac{-\lambda d v}{d x}\right)$ $=\frac{x}{v(x)} \frac{d v}{d x}=\epsilon_{v}(x)$.

Proof of Proposition 30: Consider the two consumption streams: $((0,0),(c, s),(0, s+t))$ and $((0,0),(0, s),(c, s+t))$, where $c>0$. If the consumer receives $((0,0),(0, s),(c, s+t))$ when he was expecting $((0,0),(c, s),(0, s+t))$ then, according to prospect theory, he codes the postponement of $c$ as a loss in period 2 but a gain in period 3. According to $(2.9), V_{0}((0,0),(0, s),(c, s+t))=v(0) D_{+}(0,0)+v(-c) D_{-}(0, s)+v(c) D_{+}(0, s+t)$ $=-\lambda v(c) D_{-}(0, s)+v(c) D_{+}(0, s+t)$, where the last inequality comes from $v(0)=0$ and $v(-c)=-\lambda v(c)$. On the other hand, if the consumer receives $((0,0),(c, s),(0, s+t))$ when he was expecting $((0,0),(0, s),(c, s+t))$, he codes the bringing forward of consumption as a gain in period 2 but a loss in period 3 . Hence, $V_{0}((0,0),(c, s),(0, s+t))$ 
$=v(0) D_{+}(0,0)+v(c) D_{+}(0, s)+v(-c) D_{-}(0, s+t)=v(c) D_{+}(0, s)-\lambda v(c) D_{-}(0, s+t)$.

We thus have: $V_{0}((0,0),(c, s),(0, s+t))+V_{0}((0,0),(0, s),(c, s+t))=v(c) D_{+}(0, s)-$

$\lambda v(c) D_{-}(0, s+t)-\lambda v(c) D_{-}(0, s)+v(c) D_{+}(0, s+t)=-\left[\lambda\left(D_{-}(0, s+t)+D_{-}(0, s)\right)-\left(D_{+}(0, s)+D_{+}\right.\right.$

0 , since $v(c)>0, D_{-}(0, s+t)+D_{-}(0, s) \geq D_{+}(0, s+t)+D_{+}(0, s)$ (Proposition 29) and

$\lambda>1$ (A6). Hence, $V_{0}((0,0),(c, s),(0, s+t))<-V_{0}((0,0),(0, s),(c, s+t))$.

Proof of Proposition 31: The result is obvious for the CRRA and CARA classes, from (3.1) and (3.3), respectively. We shall concentrate on giving the proof for the HARA class (3.2). For the remaining two classes: the logarithmic (3.4) and the quadratic (3.5), the proof is similar but easier and, so, will be omitted. Let $f(y)=\ln y-\gamma \ln (\mu+y)-$ $\ln \left[(\mu+y)^{1-\gamma}-\mu^{1-\gamma}\right], y=\frac{\theta x}{\gamma}, y \geq 0$. Then, from $(3.2), \epsilon_{v}(x)=(1-\gamma) e^{f\left(\frac{\theta x}{\gamma}\right)}$. Hence, $\epsilon_{v}$ is decreasing if, and only if, $f(y)$ is decreasing. Let $g(y)=\mu^{2-\gamma}+(1-\gamma) \mu^{1-\gamma} y-$ $\mu(\mu+y)^{1-\gamma}$, then it is straightforward to show that $f^{\prime}(y)<0$ if, and only if, $g(y)>0$. Simple calculations show that $g(0)=0, g^{\prime}(0)=0$ and $g^{\prime \prime}(y)=\gamma \mu(1-\gamma)(\mu+y)^{-\gamma-1}>0$. Hence, $g(y)>0$ for $y>0$. Hence $f$ and, thus, also $\epsilon_{v}$, is decreasing.

Proof of Proposition 32: Follows from (3.11) by direct calculation.

Proof of Proposition 33: These can be verified by direct calculation using (4.1), (4.2), (4.3), (4.4), (4.5) and (4.6).

\section{References}

[1] Aczel, J., 1966. Lectures on Functional Equations and their Applications. Academic Press, New York and London.

[2] al-Nowaihi, A., Dhami, S., 2006a. A note on the Loewenstein-Prelec theory of intertemporal choice. Mathematical Social Sciences 52, 99-108.

[3] al-Nowaihi, A., Dhami, S., 2006b. A simple derivation of Prelec's probability weighting function. The Journal of Mathematical Psychology 50 (6), 521-524.

[4] Andersen, S., Harrison, G. W., Lau, M. I., Rutstrom, E.E., 2007. Eliciting risk and time preferences. Forthcoming, Econometrica.

[5] Eichhorn, W., 1978. Functional Equations in Economics, Addison-Wesley.

[6] Frederick, S., Loewenstein, G., O’Donoghue, T., 2002. Time discounting and time preference: a critical review. Journal of Economic Literature 40, 351-401.

[7] Fudenberg, D., Levine, D. K., 2006. A dual-self model of impulse control. American Economic Review, 96(5) 1449-1476. 
[8] Halevy, Y., 2007. Strotz meets Allais: Diminishing impatience and the certainty effect. Forthcoming in American Economic Review.

[9] Kahneman, D., Tversky, A., 1979. Prospect theory : an analysis of decision under risk. Econometrica 47, 263-291.

[10] Kahneman D., Tversky A., 2000. Choices, Values and Frames, Cambridge University Press, Cambridge.

[11] Kahneman, D., Ritov, I., Schkade, D., 1999. Economic preferences or attitude expression? An analysis of dollar responses to public issues. Journal of Risk and Uncertainty, 19, 203-235.

[12] Laibson, D., 1997. Golden eggs and hyperbolic discounting. Quarterly Journal of Economics 112, 443-477.

[13] Loewenstein G., 1988a. Frames of mind in intertemporal choice. Management Science $34,200-214$.

[14] Loewenstein G., 1988b. The weighting of waiting: response mode effects in intertemporal choice. Working paper, Center for Decision Research, University of Chicago.

[15] Loewenstein G., Prelec D., 1992. Anomalies in intertemporal choice : evidence and an interpretation. The Quarterly Journal of Economics 107, 573-597.

[16] Luce R. D. (2001). Reduction invariance and Prelec's weighting functions. Journal of Mathematical Psychology 45, 167-179.

[17] Manzini, P. and M. Mariotti, 2006. A vague theory of choice over time. Advances in Theoretical Economics. Berkeley Electronic Press vol. 6(1), Article 6.

[18] Manzini, P. and M. Mariotti, 2008. Choice over time, in Anand, P., Pattanaik, P. and Puppe, P., The Handbook of Rational and Social Choice, Oxford, Oxford University Press.

[19] Noor, J., 2008a. Hyperbolic discounting and the standard model. Mimeo. Boston University.

[20] Noor, J., 2008b. Anomalous money-discounting. Mimeo. Boston University.

[21] Ok, E. A., and Y. Masatlioglu, 2007. A theory of (relative) discounting. Journal of Economic Theory 137, 214-245. 
[22] Phelps, E.S., Pollak, R., 1968. On second-best national saving and game-equilibrium growth. Review of Economic Studies 35, 185-199.

[23] Prelec D. (1998). The probability weighting function. Econometrica 60, 497-528.

[24] Read, D., 2001. Is time-discounting hyperbolic or subadditive? Journal of Risk and Uncertainty, 23, 5-32.

[25] Roelofsma, P. and Read, D. 2000. Intransitive intertemporal choice. Journal of Behavioral Decision Making 13, 161-177.

[26] Rubinstein, A. 2003. Is it economics and psychology? The case of hyperbolic discounting, International Economic Review, 44 (4), 1207-1216.

[27] Samuelson, P., 1937. A note on measurement of utility. Review of Economic Studies, 4, 155-161.

[28] Scholten, M. and Read, D. 2006a. Discounting by Intervals: A generalized model of intertemporal choice. Management Science, 52, 1424-1436.

[29] Scholten, M. and Read, D., 2006b. Beyond Discounting: The Tradeoff Model of Intertemporal. Choice. Working Paper LSE-OR 06.88. London: London School of Economics and Political Science.

[30] Slovic, P., 1991. The construction of preference. American Psychologist, 50, 364-371.

[31] Thaler, R.H., 1981. Some empirical evidence on dynamic consistency. Economic Letters 8, 201-207.

[32] Thaler, R.H., 1999. Mental accounting matters. Journal of Behavioral Decision Making 12, 183-206.

[33] Tversky, A., and Kahneman, D., 1991. Loss aversion in riskless choices: A referencedependent model. Quarterly Journal of Economics, 106:4, 1039-1061.

[34] Tversky, A., and Kahneman, D., 1992. Advances in prospect theory: cumulative representation of uncertainty. Journal of Risk and Uncertainty 5, 297-323. 